\title{
Volatile profile of Madeira wines submitted to traditional accelerated ageing
}

\author{
Vanda Pereira ${ }^{\mathrm{a}, \mathrm{b}, *}$, Juan Cacho $^{\mathrm{c}}$, José C. Marques ${ }^{\mathrm{a}, \mathrm{b}}$ \\ ${ }^{a}$ Centre of Exact Sciences and Engineering, University of Madeira, Campus da Penteada, 9000-390 Funchal, Portugal \\ ${ }^{\mathrm{b}}$ Institute of Nanostructures, Nanomodelling and Nanofabrication (I3N), University of Aveiro, Aveiro, Portugal \\ ${ }^{c}$ Department of Analytical Chemistry, Faculty of Sciences, University of Zaragoza, 50009 Zaragoza, Spain
}

\section{A R T I C L E I N F O}

\section{Article history:}

Received 17 December 2013

Received in revised form 8 April 2014

Accepted 9 April 2014

Available online 18 April 2014

\section{Keywords:}

Fortified wines

Accelerated ageing

Heating

Volatiles

\begin{abstract}
A B S T R A C T
The evolution of monovarietal fortified Madeira wines forced-aged by traditional thermal processing (estufagem) were studied in terms of volatiles. SPE extracts were analysed by GC-MS before and after heating at $45^{\circ} \mathrm{C}$ for 3 months (standard) and at $70^{\circ} \mathrm{C}$ for 1 month (overheating). One hundred and ninety volatile compounds were identified, 53 of which were only encountered in baked wines. Most chemical families increased after standard heating, especially furans and esters, up to 61 and 3-fold, respectively. On the contrary, alcohols, acetates and fatty acids decreased after heating. Varietal aromas, such as Malvasia's monoterpenic alcohols were not detected after baking. The accelerated ageing favoured the development of some volatiles previously reported as typical aromas of finest Madeira wines, particularly phenylacetaldeyde, $\beta$-damascenone and 5-ethoxymethylfurfural. Additionally, ethyl butyrate, ethyl 2methylbutyrate, ethyl caproate, ethyl isovalerate, guaiacol, 5-hydroxymethylfurfural and $\gamma$-decalactone were also found as potential contributors to the global aroma of baked wines.
\end{abstract}

(c) 2014 Elsevier Ltd. All rights reserved.

\section{Introduction}

Flavour is one of the most significant factors of wine quality, determining the consumer acceptance or rejection. Generally speaking, the aroma of wines is influenced by several different compounds, originated from grapes or resulting from winemaking, ageing and storage. In fact, these compounds act as a fingerprint of each type of wine. In some cases, the occurrence of a particular compound is enough to give the characteristic aroma of a wine (Polaskova, Herszage, \& Ebeler, 2008).

Madeira wine is a well-known fortified wine distinguished for its superior quality and it is characterised by marked and intense flavours (V. Pereira, 2011). Its winemaking can include a peculiar maturation process, a heating step known as estufagem. This step consists of heating the fortified wine to about $45^{\circ} \mathrm{C}$, for at least 3 months. Then, the oxidative ageing goes further, since wine is placed in wooden casks (regularly used casks), for at least 3 years. A premature ageing takes place along with heating, being acquired some of the aroma characteristics considered typical of the finest Madeiras (older wines only matured in oak casks for several years).

\footnotetext{
* Corresponding author at: Centre of Exact Sciences and Engineering, University of Madeira, Campus da Penteada, 9000-390 Funchal, Portugal. Tel.: +351 291705122 .

E-mail address: vpereira@uma.pt (V. Pereira).
}

It is well known that heating promotes important changes in the aroma of foods. Up to date, there are some studies dealing with the accelerated oxidative ageing, promoted by thermal processing, on the volatile profile of wines. Deibner and Bernard, in 1956, as referenced by Cutzach, Chatonnet, and Dubourdieu (1999), studied the effect of heat treatment on wine, pointing out the important role of Maillard reactions in the formation of the aroma of thermally processed sweet fortified wines, but at that time, they were unable to identify the respective compounds. Cutzach et al. (1999) investigated the ageing of red and white sweet fortified wines (Vins doux Naturels) following an experimental laboratory study, in which, wines were forced-aged through heating at $37^{\circ} \mathrm{C}$ for 12 months. Among the developed molecules during the accelerated ageing, the same authors found that sotolon (3hydroxy-4,5-dimethyl-2(5H)-furanone), 5-ethoxymethylfurfural, 5-hydroxymethylfurfural (HMF), furfural acetylformoin and hydroxymaltol were involved in the aroma of sweet fortified wines. Sotolon has also been considered as a key odorant of the typical aroma of oxidative aged Port wine (Silva Ferreira, Barbe, \& Bertrand, 2003). Escudero, Cacho, and Ferreira (2000) also performed studies dealing with wine oxidative ageing, through laboratory-oxidised samples at $20{ }^{\circ} \mathrm{C}$ for several weeks. According to olfactometric studies (GC-O analysis), they found that the impact odorants of oxidised white wines were essentially 2,4,5trimethyldioxolane, methional, sotolon and eugenol. Changes in 
the volatile content of Fino Sherry wines, exposed to heating $\left(45^{\circ} \mathrm{C}\right)$ and $\mathrm{UV}-\mathrm{Vis}$ radiation, have also been reported (Benítez, Castro, Natera, \& Barroso, 2006). These experiments revealed the decrease of most esters, acids and alcohols, and the increase of furfural and benzaldehyde. López de Lerma, Peinado, Moreno, and Peinado (2010) thermally processed sweet Pedro Ximénez wines at $65{ }^{\circ} \mathrm{C}$ for up to 30 days and reported the increase of volatile Maillard products, specifically of HMF, 5-ethoxymethylfurfural, dihydromaltol, 2,3-dihydro-3,5-dihydroxy-6-methyl-4H-pyran-4one (DDMP), 2-methyltetrahydrofuran-3-one, furaneol, dihydro2-methyl-3(2H)-furanone and cyclotene. Loscos, Hernández-Orte, Cacho, and Ferreira (2010) reported that accelerated ageing, at $50{ }^{\circ} \mathrm{C}$ for 9 weeks, of wines supplemented with grape flavour precursors also introduced important changes in the volatile composition. The same study showed that the main differences were observed in the first week of accelerated ageing. Most compounds first showed a significant increase and later a steady reduction, including Riesling acetal, 1,1,6-trimethyl-1,2-dihydronaphthalene (TDN), and (E)-1-(2,3,6-trimethylphenyl)buta-1,3-diene (TPB). However, vanillin derivatives, furan linalool oxides, 3-oxo- $\beta$ ionone, actinidols, 4-ethylphenol, and guaiacol showed a continuous increase during the ageing process.

Fedrizzi et al. (2011) studied the effect of accelerated ageing $\left(30^{\circ} \mathrm{C}\right.$ for 60 days) on the fermentative, varietal and sulfur compounds of a dry red Botrytis Amarone wine and found out that benzaldehyde, phenylacetaldehyde, syringaldehyde and vanillin showed a perceptible increase, while several sulfur compounds disappeared after just 15 days, under oxidative conditions. They also tentatively identified methionol-S-oxide for the first time in this kind of wine. More recently, Cejudo-Bastante, HermosínGutiérrez, and Pérez-Coello (2013) also compared accelerated ageing $\left(50^{\circ} \mathrm{C}\right.$ during 7 days) with conventional storage $\left(18^{\circ} \mathrm{C}\right.$ ) analysing the volatile composition of Chardonnay white wines. They concluded that dioxanes, dioxolanes, and TDN were developed while some alcohols, terpenes, and furanic compounds disappear with accelerated ageing. Moreover, their results demonstrated that $\beta$-damascenone and TDN levels were quite higher in the accelerated-aged wine.

In the case of Madeira wines, which are traditionally forcedaged, few studies were done regarding the effect of the baking step on their volatile profile. The first study, carried out by Oliveira e Silva et al. (2008) was based on GC-O analysis and highlighted the occurrence of particular volatiles that imparted notes considered typical of finest Madeiras bouquet. Those volatiles were identified as sotolon, furfural, 5-methylfurfural, 5-ethoxymethylfurfural, methional, and phenylacetaldehyde.

The aim of the current work is to study the impact of estufagem on the global GC-MS volatile profile of Madeira wines, focusing to a large extent on individual volatile compounds, namely some that are barely reported in Madeira wines, in order to highlight the global ageing process. For this purpose, three young Madeira wines: dry Tinta Negra (TN, red variety), sweet TN and sweet Malvasia (white variety) were heated at $45{ }^{\circ} \mathrm{C}$ for 3 months. Additionally, overheating conditions, $70^{\circ} \mathrm{C}$ for 1 month, were also studied to force the development of heating-specific volatiles.

\section{Experimental}

\subsection{Wine samples}

Three Madeira wines from two Vitis vinifera L. grape varieties of 2007 harvest were studied: dry TN, sweet TN and sweet Malvasia. The wines were produced using the winemaking practices of a Madeira wine producer. The elaboration of these wines was conducted in separated stainless steel tanks. The alcoholic fermenta- tion was conducted under controlled temperature and without adding any commercial yeast. The fermentation for TN sweet wine was stopped adding alcohol $(95 \% \mathrm{v} / \mathrm{v})$ when the must specific gravity attained $1.025 \mathrm{~g} / \mathrm{mL}$, which corresponded to $115 \mathrm{~g} / \mathrm{L}$ of reducing sugars in the wine, while for TN dry it was allowed to reach a specific gravity of $0.986 \mathrm{~g} / \mathrm{mL}$ before fortification, allowing a low level of residual sugars in the wine (about $4 \mathrm{~g} / \mathrm{L}$ ). Malvasia sweet wine must was fermented up to $1.019 \mathrm{~g} / \mathrm{mL}$, keeping the reducing sugars at $96 \mathrm{~g} / \mathrm{L}$. After post-fermentation treatments, each wine was heated at $45^{\circ} \mathrm{C}$ for 3 months in a special pilot scale system, equipped with 200 -L stainless steel tanks, fitted with heat coils that allow hot water to circulate inside the container. To force the development of volatiles specific from overheating, each wine was also submitted to $70^{\circ} \mathrm{C}$ for 1 month (overheating conditions). All samples were kept at $-20^{\circ} \mathrm{C}$ before being analysed.

\subsection{Chemicals}

All reagents were of analytical grade. Dichloromethane HPLCgrade was from Fisher Scientific (Loughborough, UK) while absolute ethanol was supplied by Sigma-Aldrich (St. Louis, MO). Ultra-pure water was obtained from a Milli-Q system (Millipore, Milford, MA). Solid anhydrous sodium sulfate was from JMGS (Lisbon, Portugal) while the 3-octanol standard was from SigmaAldrich. LiChrolut EN resin was supplied by Merck Co. (Darmstadt, Germany), while the 6-mL polypropylene cartridges and respective frits were obtained from Supelco (Bellefonte, PA). Solid-phase extraction was carried out in a 12-port Visiprep ${ }^{\mathrm{TM}}$ SPE vacuum manifold from Supelco.

\subsection{Sample extraction}

The screening of volatiles was accomplished based on the solidphase extraction (SPE) method proposed by López, Aznar, Cacho, and Ferreira (2002). Briefly, $120 \mathrm{mg}$ of LiChrolut EN resin were packed in a 6-mL cartridge. After conditioning the resin, in the SPE station, with $4 \mathrm{~mL}$ of dichloromethane, $4 \mathrm{~mL}$ of methanol and $4 \mathrm{~mL}$ of ethanolic solution $(18 \%, \mathrm{v} / \mathrm{v}), 50 \mathrm{~mL}$ of wine spiked with $25 \mu \mathrm{L}$ of 3 -octanol $(491 \mathrm{mg} / \mathrm{L})$ were passed through the resin at about $2 \mathrm{~mL} / \mathrm{min}$. Then, the sorbent was dried by passing a small flow of air through it for $15 \mathrm{~min}$. Finally, wine volatiles were eluted with $1.3 \mathrm{~mL}$ of dichloromethane and the extract dried with sodium sulfate, being kept at $-20^{\circ} \mathrm{C}$ until analysis. All samples were extracted in duplicate.

\subsection{GC-MS analysis}

The extracts were analysed using a TRACE GC Ultra gas chromatograph equipped with the ISQ single quadrupole (electron impact mode) and the TriPlus autosampler (liquid mode) from Thermo Scientific (Hudson, $\mathrm{NH}$ ). One microlitre of extract was vaporised in the injector port at $230{ }^{\circ} \mathrm{C}$ in splitless mode $(1 \mathrm{~min})$. All extracts were injected twice. The column was a DB-WAXetr $30 \mathrm{~m} \times 0.32 \mathrm{~mm}$ with $0.5 \mu \mathrm{m}$ film thickness from Agilent $\mathrm{J} \& \mathrm{~W}$ (Folsom, CA) and the carrier gas was He at $1 \mathrm{~mL} / \mathrm{min}$. The ionisation voltage was $70 \mathrm{eV}$ with transfer line and ion source temperatures kept at 230 and $240{ }^{\circ} \mathrm{C}$, respectively. The oven temperature program started at $40^{\circ} \mathrm{C}$ for $5 \mathrm{~min}$ then increased up to $230^{\circ} \mathrm{C}$ at $3{ }^{\circ} \mathrm{C} / \mathrm{min}$ and finally was kept at $230^{\circ} \mathrm{C}$ for $15 \mathrm{~min}$. The $\mathrm{m} / \mathrm{z} 30$ 400 mass range was recorded.

The identification of compounds was made by comparison of the mass spectra obtained with those present in NIST08 and Wiley 6.0 MS library databases, and comparing the obtained Kovats indexes with those stated on NIST Chemistry WebBook (Stein, 2008). The compounds, namely those that were only identified comparing the obtained mass spectra with those present in the 
MS library databases, were just considered when a fair match was achieved (>70\%). A $C_{7}-C_{30} n$-alkanes mixture (Supelco) was used to calculate the Kovats indexes. 3-Octanol was added to each sample as internal standard (IS). The amount of each volatile compound was estimated, semi-quantitatively, regarding the added amount of IS $(246 \mu \mathrm{g} / \mathrm{L})$ and the relative concentrations of the investigated compounds were measured as follows:

Semi-quantitative concentrations $=($ peak area/IS peak area $) \times$ IS concentration. The coefficient of variation (\% CV) was on average $7 \%$.

\section{Results and discussion}

Typical chromatograms of the current Madeira wine extracts are depicted in Fig. 1. Table 1 summarises the data of the volatile compounds encountered in the studied Madeira wines, before and after the heating at standard $\left(45^{\circ} \mathrm{C}\right.$ for 3 months $)$ and overheating $\left(70^{\circ} \mathrm{C}\right.$ for 1 month) conditions. The concentrations given in Table 1 are rough estimates, but still give an idea of which order of magnitude the compounds are sensorially active and information about the evolution of each compound with heating.

The GC-MS analyses of the current sample set allowed the identification of 190 volatile compounds, including 42 esters, 29 alcohols, 18 carbonyl compounds, 19 volatile phenols, 17 fatty acids, 15 furan compounds, 15 monoterpenes, 8 acetals, 7 lactones, 4 sulfur compounds, 6 norisoprenoids and 10 other compounds. At least 171 other compounds could not be identified by the regular strategies and will require specific methods of isolation and characterisation.

The current study also revealed that a large number of compounds were developed during the traditional heating of Madeira wines (Fig. 2); 53 of the identified compounds were exclusively found in baked wines. The results showed that young TN dry wine presented the highest fraction of volatiles, about $52 \mathrm{mg} / \mathrm{L}$ (before heating), which is in agreement with the fermentation degree, essentially due to the higher levels of esters and higher alcohols
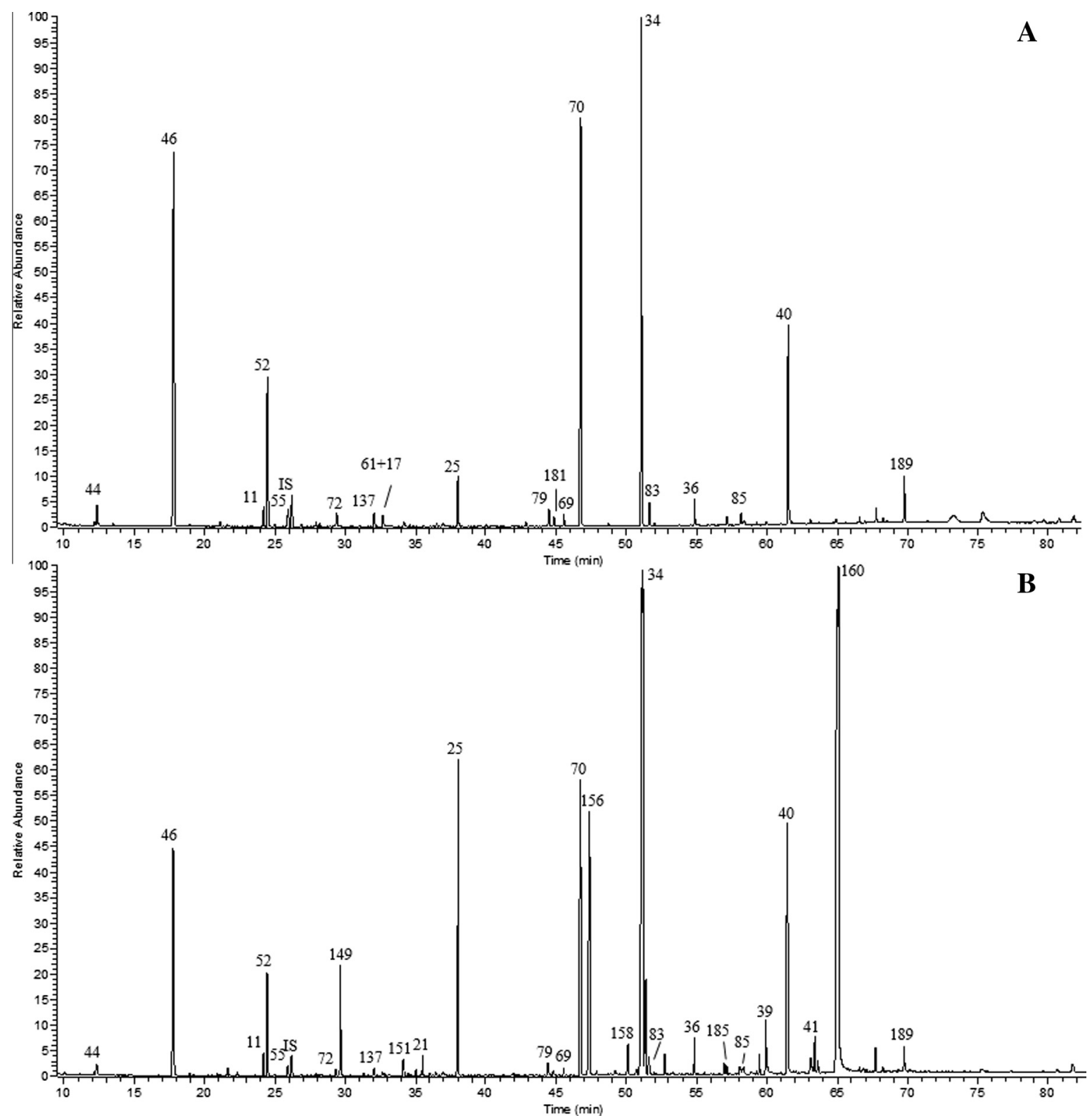

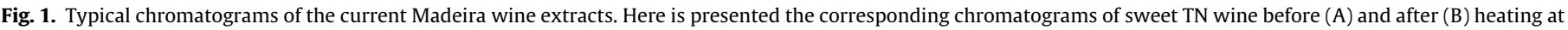
$70{ }^{\circ} \mathrm{C}$ for 1 month. For peak identification see Table 1 . Only the major peaks are highlighted. 
Table 1

Volatile compounds identified in TN sweet, TN dry and Malvasia wines before and after the heating at $45^{\circ} \mathrm{C}$ ( 3 months) and $70{ }^{\circ} \mathrm{C}(1$ month).

\begin{tabular}{|c|c|c|c|c|c|c|c|c|c|c|c|c|c|c|c|c|c|c|c|c|c|c|}
\hline \multirow[t]{2}{*}{ \# } & \multirow[t]{2}{*}{ Compounds } & \multirow[t]{2}{*}{$\mathrm{KI}$} & \multicolumn{6}{|c|}{ TN sweet $(\mu \mathrm{g} / \mathrm{L})$} & \multicolumn{6}{|c|}{ TN dry $(\mu \mathrm{g} / \mathrm{L})$} & \multicolumn{6}{|c|}{ Malvasia $(\mu \mathrm{g} / \mathrm{L})$} & \multirow{2}{*}{$\begin{array}{l}\text { Common } \\
\text { descriptors }^{\mathrm{a}}\end{array}$} & \\
\hline & & & $0 \mathrm{~m}$ & $\pm \mathrm{SD}$ & $\begin{array}{l}3 \mathrm{~m} \\
45^{\circ} \mathrm{C}\end{array}$ & $\pm \mathrm{SD}$ & $\begin{array}{l}1 \mathrm{~m} \\
70^{\circ} \mathrm{C}\end{array}$ & $\pm \mathrm{SD}$ & $0 \mathrm{~m}$ & $\pm \mathrm{SD}$ & $\begin{array}{l}3 \mathrm{~m} \\
45^{\circ} \mathrm{C}\end{array}$ & $\pm \mathrm{SD}$ & $\begin{array}{l}1 \mathrm{~m} \\
70^{\circ} \mathrm{C}\end{array}$ & $\pm \mathrm{SD}$ & $0 \mathrm{~m}$ & $\pm \mathrm{SD}$ & $\begin{array}{l}3 \mathrm{~m} \\
45^{\circ} \mathrm{C}\end{array}$ & $\pm \mathrm{SD}$ & $\begin{array}{l}1 \mathrm{~m} \\
70^{\circ} \mathrm{C}\end{array}$ & $\pm \mathrm{SD}$ & & \\
\hline & Esters (42) & & & & & & & & & & & & & & & & & & & & & \\
\hline 1 & Ethyl butyrate & 1053 & 6.2 & 0.2 & 9.5 & 0.5 & 11.5 & 0.5 & 19.2 & 1.3 & 27.5 & 0.6 & 52.7 & 20.8 & 4.8 & 0.3 & 7.8 & 0.1 & 9.5 & 0.3 & Fruity & \\
\hline 2 & Monomethyl succinate & 1062 & n.d. & & n.d. & & n.d. & & n.d. & & n.d. & & 28.6 & 8.7 & n.d. & & n.d. & & n.d. & & & \\
\hline 3 & Ethyl methylbutyrate ${ }^{\omega *}$ & 1069 & n.d. & & n.d. & & n.d. & & n.d. & & 8.4 & 0.4 & 25.3 & 8.5 & n.d. & & n.d. & & 6.7 & 1.0 & Fruity & \\
\hline 4 & Ethyl isovalerate & 1085 & n.d. & & n.d. & & 6.9 & 1.0 & n.d. & & 14.1 & 0.8 & 47.5 & 18.4 & n.d. & & 4.3 & 0.4 & 9.2 & 1.3 & Fruity, apple & \\
\hline 5 & Isoamyl acetate" & 1141 & 10.6 & 0.3 & 3.1 & 0.5 & n.d. & & 91.7 & 5.0 & 19.8 & 1.9 & 22.2 & 0.2 & 16.1 & 1.5 & 4.3 & 0.6 & n.d. & & Banana & \\
\hline 6 & Ethyl caproate ${ }^{*}$ & 1251 & 25.7 & 0.5 & 30.0 & 1.7 & 37.4 & 2.6 & 96.6 & 5.4 & 96.4 & 2.4 & 150.8 & 3.5 & 33.0 & 3.5 & 43.8 & 0.6 & 48.9 & 1.7 & Fruity & \\
\hline 7 & Hexyl acetate" & 1289 & 2.9 & 0.2 & n.d. & & n.d. & & 6.7 & 0.6 & n.d. & & n.d. & & 3.0 & 0.4 & 0.0 & 0.0 & n.d. & & Fruity, pear & \\
\hline 8 & Ethyl pyruvate & 1293 & 10.9 & 0.2 & 10.8 & 0.6 & 28.3 & 0.9 & 26.4 & 0.9 & 27.9 & 0.8 & 20.7 & 1.4 & 34.4 & 0.1 & 26.6 & 1.0 & 39.0 & 1.1 & $\begin{array}{l}\text { Herbaceous, oil } \\
\text { painting }\end{array}$ & \\
\hline 9 & Ethyl 3-hexenoate" & 1320 & n.d. & & n.d. & & n.d. & & n.d. & & 2.8 & 0.5 & n.d. & & 3.1 & 0.3 & 3.7 & 0.4 & 3.7 & 0.3 & - & \\
\hline 10 & Ethyl 3-ethoxypropionate" & 1354 & n.d. & & n.d. & & 7.9 & 0.3 & n.d. & & n.d. & & n.d. & & n.d. & & 1.8 & 0.3 & 8.1 & 0.3 & - & \\
\hline 11 & Ethyl lactate & 1368 & 168.2 & 3.2 & 251.3 & 3.0 & 298.5 & 3.8 & 1033.7 & 15.2 & 1349.8 & 29.3 & 1228.0 & 34.5 & 290.1 & 5.8 & 381.8 & 4.7 & 447.4 & 9.6 & $\begin{array}{l}\text { Strawberry, } \\
\text { raspberry }\end{array}$ & \\
\hline 12 & Ethyl glycolate & 1445 & n.d. & & 5.6 & 0.4 & 9.2 & 0.3 & n.d. & & 4.5 & 0.4 & 4.6 & 0.4 & 46.8 & 4.2 & 12.2 & 0.8 & 11.2 & 0.7 & - & \\
\hline 13 & Ethyl 2-hydroxyisovalerate & 1450 & 2.2 & 0.1 & 6.2 & 0.1 & 9.7 & 0.2 & 11.3 & 0.7 & 34.4 & 1.8 & 52.1 & 0.2 & n.d. & & 21.1 & 0.3 & 30.3 & 0.8 & - & \\
\hline 14 & Ethyl caprylate & 1452 & 14.3 & 0.4 & 12.0 & 0.7 & 14.0 & 1.3 & 95.9 & 3.4 & 57.8 & 1.3 & 92.0 & 2.2 & 20.1 & 2.5 & 30.5 & 0.6 & 20.6 & 0.6 & Fruity, apple & $<$ \\
\hline 15 & Isobutyl lactate & 1483 & n.d. & & n.d. & & n.d. & & 12.9 & 0.7 & 32.3 & 2.3 & 23.3 & 2.9 & n.d. & & n.d. & & n.d. & & - & $\ddot{8}$ \\
\hline 16 & Ethyl 3-hydroxybutyrate" & 1544 & n.d. & & 3.3 & 0.2 & 4.1 & 0.2 & 13.0 & 0.4 & 14.0 & 0.8 & 14.3 & 1.0 & n.d. & & n.d. & & n.d. & & - & $\frac{0}{0}$ \\
\hline 17 & Ethyl leucate & 1568 & 9.1 & 0.1 & 15.0 & 0.1 & 18.3 & 0.4 & 46.5 & 0.8 & 83.6 & 1.6 & 95.8 & 1.4 & 24.0 & 0.2 & 32.6 & 0.8 & 42.5 & 0.3 & - & . \\
\hline 18 & Ethyl 4-oxobutyrate ${ }^{* * * *}$ & 1576 & 5.5 & 0.1 & 7.1 & 0.0 & 9.4 & 0.5 & 3.3 & 0.3 & 5.2 & 0.3 & 4.6 & 0.1 & 2.5 & 0.2 & 5.3 & 0.2 & 5.8 & 0.8 & - & $\stackrel{2}{2}$ \\
\hline 19 & Isoamyl lactate & 1593 & n.d. & & n.d. & & n.d. & & 37.7 & 0.6 & 49.6 & 1.1 & 60.3 & 2.9 & n.d. & & 4.3 & 0.5 & 4.7 & 0.3 & - & $\vec{E}$ \\
\hline 20 & Diethyl malonate ${ }^{* *}$ & 1602 & 4.6 & 0.1 & 5.4 & 0.1 & 2.5 & 0.1 & n.d. & & n.d. & & n.d. & & 2.0 & 0.1 & 6.9 & 0.1 & n.d. & & - & $=$ \\
\hline 21 & Ethyl levulinate ${ }^{* * *}$ & 1636 & n.d. & & 6.4 & 0.0 & 131.7 & 0.8 & n.d. & & 7.6 & 0.1 & 24.8 & 1.4 & 1.1 & 0.1 & 9.4 & 0.1 & 145.3 & 2.2 & - & 8 \\
\hline 22 & Ethyl caprate & 1655 & 5.9 & 0.2 & 4.8 & 0.2 & 3.6 & 0.4 & 92.8 & 2.2 & 36.3 & 3.0 & 30.1 & 0.6 & 6.5 & 1.2 & 7.0 & 0.5 & 3.5 & 0.3 & Grape & $5^{0}$ \\
\hline 23 & Ethyl methyl succinate ${ }^{* * *}$ & 1662 & n.d. & & n.d. & & n.d. & & n.d. & & n.d. & & n.d. & & 2.6 & 0.1 & 9.1 & 0.2 & 13.4 & 0.3 & - & $\frac{0}{0}$ \\
\hline 24 & Diethyl methylsuccinate ${ }^{\cdots *}$ & 1665 & n.d. & & 3.4 & 0.3 & 12.1 & 1.2 & n.d. & & 8.7 & 1.0 & 26.0 & 0.4 & n.d. & & n.d. & & n.d. & & - & క్n̆. \\
\hline 25 & Diethyl succinate & 1699 & 253.3 & 4.6 & 1627.4 & 13.3 & 2643.4 & 74.3 & 1277.8 & 14.9 & 4677.0 & 49.0 & 6240.2 & 988.7 & 691.2 & 6.9 & 3170.3 & 33.9 & 4742.7 & 20.1 & Fruity, wine & $\frac{5}{3}$ \\
\hline 26 & Ethyl 9-decenoate & 1708 & n.d. & & n.d. & & n.d. & & 6.1 & 0.6 & n.d. & & n.d. & & n.d. & & n.d. & & n.d. & & - & \\
\hline 27 & Diethyl glutarate & 1803 & 1.3 & 0.0 & 5.2 & 0.0 & 7.6 & 0.3 & 4.1 & 0.1 & 17.7 & 0.2 & 23.4 & 0.8 & 2.4 & 0.0 & 9.2 & 0.0 & 12.5 & 0.3 & - & $\bar{N}$ \\
\hline 28 & Ethyl phenylacetate & 1812 & 4.4 & 0.5 & 8.0 & 0.5 & 9.5 & 0.3 & 11.9 & 0.5 & 22.7 & 1.0 & 15.7 & 1.0 & 13.9 & 0.6 & 19.2 & 1.0 & 30.1 & 2.3 & $\begin{array}{l}\text { Fruity, sweet, } \\
\text { honey-like }\end{array}$ & $\widetilde{\widetilde{O}}$ \\
\hline 29 & Ethyl 4-hydroxybutyrate"* & 1834 & 11.5 & 1.1 & 23.0 & 2.7 & 26.2 & 1.9 & 159.5 & 6.8 & 74.2 & 2.4 & 51.8 & 3.2 & 38.4 & 1.4 & 3.6 & 0.4 & 26.2 & 0.3 & & \pm \\
\hline 30 & Phenylethyl acetate & 1844 & 7.8 & 0.1 & n.d. & & n.d. & & 39.0 & 0.7 & 11.7 & 0.5 & 10.4 & 0.3 & 14.9 & 0.3 & 3.7 & 0.2 & n.d. & & $\begin{array}{l}\text { Roses, apple, } \\
\text { honey, sweet }\end{array}$ & $\underset{\sim}{\tilde{N}}$ \\
\hline 31 & Ethyl laurate ${ }^{* *}$ & 1858 & 5.6 & 0.8 & n.d. & & n.d. & & 12.2 & 1.1 & n.d. & & n.d. & & 4.6 & 0.9 & n.d. & & n.d. & & $\begin{array}{l}\text { Sweet, floral, } \\
\text { fruity, cream }\end{array}$ & $\underset{\omega}{\grave{\omega}}$ \\
\hline 32 & Diethyl adipate $^{* \omega *}$ & 1921 & n.d. & & n.d. & & n.d. & & 4.7 & 0.7 & 4.0 & 0.5 & n.d. & & n.d. & & 3.9 & 0.4 & 6.3 & 0.4 & - & \\
\hline 33 & $\begin{array}{l}\text { Ethyl 3-methylbutyl } \\
\text { butanedioate }\end{array}$ & 1925 & 3.3 & 0.4 & 7.7 & 0.3 & 7.4 & 0.7 & 28.2 & 1.6 & 46.6 & 1.8 & 75.5 & 4.5 & n.d. & & 9.0 & 0.7 & 15.9 & 1.4 & - & \\
\hline 34 & Diethyl malate & 2077 & 4824.1 & 50.1 & 13657.0 & 815.7 & 16017.9 & 1186.2 & 3836.4 & 150.2 & 11597.3 & 210.8 & 13007.9 & 1967.0 & 4935.0 & 264.9 & 13125.3 & 291.4 & 15906.6 & 51.8 & $\begin{array}{l}\text { Over-ripe, peach, } \\
\text { cut grass }\end{array}$ & \\
\hline 35 & $\begin{array}{l}\text { Propyl ethyl } \\
\text { hydroxybutanedioate }\end{array}$ & 2159 & n.d. & & n.d. & & n.d. & & n.d. & & n.d. & & 12.5 & 0.9 & n.d. & & n.d. & & n.d. & & - & \\
\hline 36 & Diethyl 2-hydroxyglutarate & 2196 & 232.7 & 4.0 & 524.1 & 12.1 & 486.8 & 18.5 & 1037.1 & 45.1 & 2208.5 & 89.4 & 2127.1 & 74.6 & 420.9 & 4.2 & 900.5 & 14.6 & 849.6 & 13.5 & Cotton candy & \\
\hline 37 & Ethyl palmitate & 2268 & n.d. & & n.d. & & n.d. & & n.d. & & n.d. & & n.d. & & 11.7 & 2.1 & n.d. & & n.d. & & - & \\
\hline 38 & Ethyl phenyl lactate & 2314 & 52.5 & 1.7 & 90.2 & 3.2 & 99.3 & 6.5 & 408.3 & 17.7 & 534.6 & 16.2 & 529.7 & 23.9 & 137.7 & 3.3 & 242.4 & 7.1 & 313.4 & 5.1 & - & \\
\hline 39 & Diethyl tartrate & 2371 & 49.5 & 3.7 & 406.5 & 7.5 & 947.9 & 30.8 & 70.7 & 7.4 & 330.5 & 24.2 & 759.9 & 17.4 & 38.6 & 7.3 & 409.1 & 27.2 & 1139.4 & 30.8 & Earth, must & \\
\hline 40 & Ethyl hydrogen succinate & 2426 & 1928.7 & 25.8 & 3997.2 & 241.7 & 3620.3 & 229.2 & 6723.4 & 880.5 & 8836.9 & 236.3 & 8001.3 & 1172.6 & 4354.1 & 239.0 & 6487.2 & 106.9 & 6435.1 & 83.7 & Sweet, sour, fruity & \\
\hline 41 & Ethyl citrate & 2499 & n.d. & & 46.3 & 0.3 & 239.8 & 12.0 & n.d. & 0.0 & 59.0 & 3.0 & 239.7 & 12.0 & n.d. & & 92.2 & 16.1 & 219.6 & 9.9 & - & \\
\hline 42 & Ethyl 4-hydroxyphenylacetate ${ }^{* \ldots *}$ & 2958 & n.d. & & n.d. & & n.d. & & 24.6 & 1.9 & 39.4 & 5.6 & 42.9 & 1.5 & 31.3 & 2.0 & 42.9 & 7.4 & 43.7 & 3.7 & - & \\
\hline & Higher alcohols (29) & & & & & & & & & & & & & & & & & & & & & \\
\hline 43 & 1-Propanol ${ }^{*}$ & 1056 & 7.1 & 0.6 & 7.2 & 0.8 & 7.7 & 0.6 & 49.1 & 6.7 & 81.0 & 28.2 & 52.6 & 17.9 & 19.1 & 0.6 & 16.7 & 1.1 & 22.5 & 1.2 & Fresh, alcohol & \\
\hline 44 & Isobutyl alcohol" & 1108 & 234.7 & 4.7 & 224.8 & 3.2 & 196.0 & 12.5 & 1227.6 & 36.6 & 1337.7 & 187.8 & 998.5 & 44.8 & 441.3 & 18.9 & 403.3 & 5.9 & 412.1 & 11.1 & Fusel, alcohol & \\
\hline 45 & 1-Butanol ${ }^{*}$ & 1163 & 5.8 & 0.4 & 6.9 & 0.5 & 4.6 & 0.6 & 21.3 & 1.6 & 22.0 & 2.0 & 15.9 & 1.3 & 5.6 & 0.6 & 12.8 & 0.7 & 7.1 & 0.5 & Medicinal, alcohol & \\
\hline 46 & Active amyl alcohol"* & 1228 & 4181.4 & 93.0 & 4335.6 & 198.2 & 3679.7 & 168.7 & 16556.2 & 1580.6 & 15336.1 & 353.7 & 13465.9 & 1512.1 & 7448.5 & 578.4 & 6937.7 & 170.9 & 6988.2 & 251.6 & Fusel & \\
\hline
\end{tabular}

(continued on next page) 


\begin{tabular}{|c|c|c|c|c|c|c|c|c|c|c|c|c|c|c|c|c|c|c|c|c|c|}
\hline \multirow[t]{2}{*}{$\#$} & \multirow[t]{2}{*}{ Compounds } & \multirow[t]{2}{*}{ KI } & \multicolumn{6}{|c|}{ TN sweet $(\mu \mathrm{g} / \mathrm{L})$} & \multicolumn{6}{|c|}{$\underline{\text { TN dry }(\mu \mathrm{g} / \mathrm{L})}$} & \multicolumn{6}{|c|}{$\underline{\text { Malvasia }(\mu \mathrm{g} / \mathrm{L})}$} & \multirow{2}{*}{$\begin{array}{l}\text { Common } \\
\text { descriptors }^{\mathrm{a}}\end{array}$} \\
\hline & & & $0 \mathrm{~m}$ & $\pm \mathrm{SD}$ & $\begin{array}{l}3 \mathrm{~m}, \\
45{ }^{\circ} \mathrm{C}\end{array}$ & $\pm \mathrm{SD}$ & $\begin{array}{l}1 \mathrm{~m}, \\
70^{\circ} \mathrm{C}\end{array}$ & $\pm \mathrm{SD}$ & $0 \mathrm{~m}$ & $\pm \mathrm{SD}$ & $\begin{array}{l}3 \mathrm{~m}, \\
45^{\circ} \mathrm{C}\end{array}$ & $\pm \mathrm{SD}$ & $\begin{array}{l}1 \mathrm{~m}, \\
70^{\circ} \mathrm{C}\end{array}$ & $\pm \mathrm{SD}$ & $0 \mathrm{~m}$ & $\pm \mathrm{SD}$ & $\begin{array}{l}3 \mathrm{~m}, \\
45^{\circ} \mathrm{C}\end{array}$ & $\pm \mathrm{SD}$ & $\begin{array}{l}1 \mathrm{~m}, \\
70^{\circ} \mathrm{C}\end{array}$ & $\pm \mathrm{SD}$ & \\
\hline 47 & 1-Pentanol ${ }^{* *}$ & 1270 & 4.8 & 0.4 & 4.1 & 0.5 & 2.7 & 0.1 & 13.2 & 0.7 & 9.6 & 0.5 & 6.6 & 0.2 & 6.9 & 0.9 & 6.1 & 0.4 & 5.2 & 0.3 & Fruity, balsamic \\
\hline 48 & 4-Methyl-1-pentanol "* & 1334 & 3.0 & 0.2 & 3.3 & 0.1 & 3.9 & 0.5 & 13.5 & 0.9 & 13.7 & 0.5 & 15.9 & 0.5 & 5.7 & 0.4 & 4.6 & 0.3 & 6.1 & 0.4 & Almond, toasted \\
\hline 49 & 2-Heptanol ${ }^{* *}$ & 1339 & 1.8 & 0.1 & 1.7 & 0.0 & 1.7 & 0.1 & 2.3 & 0.1 & 2.4 & 0.1 & 2.5 & 0.1 & n.d. & & n.d. & & n.d. & & $\begin{array}{l}\text { Fruity, mouldy, } \\
\text { musty }\end{array}$ \\
\hline 50 & (Z)-2-penten-1-ol "** & 1343 & 2.1 & 0.3 & 1.8 & 0.3 & 2.3 & 0.3 & 2.7 & 0.4 & 2.9 & 0.5 & 2.9 & 0.4 & 3.6 & 0.6 & 3.3 & 0.6 & 3.6 & 0.5 & - \\
\hline 51 & 3-Methyl-1-pentanol ${ }^{* *}$ & 1348 & 4.0 & 0.3 & 3.6 & 0.3 & 4.5 & 0.5 & 19.3 & 0.3 & 19.1 & 0.7 & 19.5 & 0.3 & 7.7 & 0.6 & 6.8 & 0.7 & 7.9 & 0.6 & - \\
\hline 52 & 1-Hexanol ${ }^{*+*}$ & 1374 & 1247.5 & 13.9 & 1268.4 & 23.6 & 1255.3 & 27.6 & 1088.4 & 8.1 & 1054.1 & 14.7 & 1090.7 & 10.0 & 1117.6 & 36.6 & 992.8 & 11.1 & 1058.9 & 10.5 & Green, grass \\
\hline 53 & $(E)$-3-hexen-1-ol ${ }^{* *}$ & 1385 & 22.6 & 0.9 & 21.7 & 0.6 & 22.2 & 1.2 & 17.6 & 0.2 & 19.8 & 0.7 & 17.3 & 0.4 & 21.6 & 0.7 & 25.2 & 0.5 & 22.3 & 0.3 & Green, floral \\
\hline 54 & 3-Ethoxy-1-propanol " & 1396 & 2.1 & 0.3 & 1.7 & 0.1 & n.d. & & 14.7 & 0.4 & 13.5 & 0.8 & 11.7 & 0.3 & 6.0 & 0.3 & 5.3 & 0.2 & 6.2 & 0.4 & Fruity \\
\hline 55 & (Z)-3-hexen-1-ol & 1405 & 150.9 & 2.6 & 142.6 & 1.9 & 132.0 & 0.7 & 164.0 & 1.3 & 147.0 & 4.0 & 142.4 & 1.5 & 291.6 & 3.7 & 253.1 & 1.5 & 251.0 & 4.8 & Grass, green \\
\hline 56 & (E)-2-hexen-1-ol ${ }^{* *}$ & 1429 & 18.4 & 0.4 & 18.1 & 0.1 & 20.5 & 0.7 & 3.2 & 0.1 & 3.8 & 0.5 & 4.7 & 0.5 & 17.7 & 0.2 & 17.0 & 0.3 & 17.1 & 0.4 & Grass, herb \\
\hline 57 & (Z)-2-hexen-1-ol" & 1439 & 7.3 & 0.7 & 7.3 & 0.5 & 8.0 & 0.3 & 4.1 & 0.3 & 4.9 & 0.7 & 5.1 & 0.4 & 5.0 & 0.7 & 4.9 & 0.6 & 4.2 & 0.5 & Grass, herb \\
\hline 58 & 1-Heptanol ${ }^{* *}$ & 1476 & 4.8 & 0.3 & 4.3 & 0.1 & 4.5 & 0.3 & 9.9 & 0.1 & 9.7 & 0.5 & 10.6 & 0.3 & 5.9 & 0.5 & 4.9 & 0.2 & 5.9 & 0.2 & Grape, sweet \\
\hline 59 & 2-Ethyl-1-hexanol" & 1510 & 5.0 & 0.2 & 8.7 & 0.2 & 4.1 & 0.1 & 5.8 & 0.2 & 13.7 & 0.3 & 8.4 & 0.6 & 5.4 & 0.6 & 10.5 & 0.6 & 4.6 & 0.3 & $\begin{array}{l}\text { Mushroom, sweet } \\
\text { fruit }\end{array}$ \\
\hline 60 & 3,5,5-Trimethylhexanol ${ }^{* * *}$ & 1527 & 2.9 & 0.1 & 3.4 & 0.4 & 4.3 & 0.2 & 6.7 & 0.4 & 6.7 & 0.5 & 9.1 & 0.5 & n.d. & & n.d. & & 4.1 & 0.2 & - \\
\hline 61 & 2,3-Butanediol, isomer 1" & 1566 & 81.0 & 1.4 & 69.7 & 16.1 & 28.5 & 6.5 & 173.7 & 72.2 & 267.2 & 111.4 & 255.2 & 4.3 & n.d. & & n.d. & & n.d. & & Butter, cream \\
\hline 62 & 1-Octanol ${ }^{*}$ & 1578 & 8.2 & 0.9 & 8.3 & 0.9 & 9.3 & 0.6 & 14.4 & 1.4 & 12.8 & 0.9 & 11.6 & 1.9 & 16.4 & 2.1 & 9.3 & 0.7 & 7.7 & 1.3 & Citrus, roses \\
\hline 63 & 2,3-Butanediol, isomer 2 & 1602 & n.d. & & n.d. & & n.d. & & 28.7 & 14.3 & 50.1 & 25.6 & 43.8 & 1.9 & n.d. & & n.d. & & n.d. & & - \\
\hline 64 & 1,2-Propanediol & 1619 & 3.9 & 0.4 & n.d. & & n.d. & & 4.1 & 1.4 & 10.9 & 5.4 & 8.3 & 1.4 & n.d. & & n.d. & & n.d. & & - \\
\hline 65 & 1-Nonanol ${ }^{* *}$ & 1680 & 1.6 & 0.1 & 1.7 & 0.1 & 2.0 & 0.1 & 4.0 & 0.1 & 4.1 & 0.2 & 4.4 & 0.1 & 2.9 & 0.0 & 2.7 & 0.1 & n.d. & & Fat, green \\
\hline 66 & 1-Methoxy-2-butanol ${ }^{-\ldots *}$ & 1683 & n.d. & & n.d. & & n.d. & & 8.5 & 2.5 & 10.1 & 2.5 & 10.3 & 0.3 & n.d. & & n.d. & & n.d. & & - \\
\hline 67 & 1-Decanol" & 1783 & n.d. & & n.d. & & n.d. & & 2.6 & 0.2 & 2.7 & 0.6 & 5.4 & 0.9 & 2.7 & 0.2 & n.d. & & n.d. & & $\begin{array}{l}\text { Orange flowery, } \\
\text { fatty }\end{array}$ \\
\hline 68 & Buthoxyethoxyethanol"** & 1819 & 35.1 & 0.9 & 7.0 & 0.5 & 8.7 & 0.8 & 6.9 & 0.6 & n.d. & & n.d. & & 12.9 & 1.6 & 12.3 & 1.1 & 13.3 & 1.8 & - \\
\hline 69 & Benzyl alcohol ${ }^{*}$ & 1909 & 120.7 & 2.5 & 111.5 & 0.7 & 114.5 & 5.6 & 219.8 & 6.9 & 198.2 & 9.8 & 198.8 & 4.5 & 117.0 & 1.7 & 115.3 & 2.3 & 128.4 & 2.5 & $\begin{array}{l}\text { Citrusy, sweet, } \\
\text { almonds }\end{array}$ \\
\hline 70 & Phenylethyl alcohol ${ }^{\circ}$ & 1945 & 3782.2 & 41.9 & 4148.3 & 88.5 & 4062.6 & 130.6 & 13672.5 & 2289.4 & 12982.1 & 360.7 & 12244.3 & 2053.4 & 8027.4 & 648.7 & $7,832.3$ & 149.9 & 8263.5 & 114.1 & $\begin{array}{l}\text { Flowery, pollen, } \\
\text { perfumed, roses }\end{array}$ \\
\hline 71 & 2-Ethoxybenzyl alcohol ${ }^{* *}$ & 1978 & n.d. & & 25.3 & 0.3 & 62.0 & 0.2 & n.d. & & 30.0 & 1.7 & 49.9 & 1.4 & n.d. & & 32.0 & 2.4 & 108.9 & 5.1 & - \\
\hline & $\begin{array}{l}\text { Fatty acids (17) } \\
\text { Acetic acid" }\end{array}$ & 1488 & 106.9 & 5.0 & & 16.8 & 67.6 & 13.5 & & 30.9 & & & & & & & & & & & \\
\hline 72 & $\begin{array}{l}\text { Acetic acid" } \\
\text { Propionic acid"* }\end{array}$ & $\begin{array}{l}1488 \\
1573\end{array}$ & 100.9 & 3.0 & $\begin{array}{r}108.5 \\
\text { n.d. }\end{array}$ & 16.8 & $\begin{array}{r}6.6 \\
2.5\end{array}$ & $\begin{array}{r}13.5 \\
0.2\end{array}$ & $\begin{array}{r}88.4 \\
55\end{array}$ & $\begin{array}{r}0.9 \\
30.8\end{array}$ & $\begin{array}{r}156.1 \\
38\end{array}$ & $\begin{array}{r}76.9 \\
0.4\end{array}$ & 138.3 & 7.1 & 55.0 & 4.6 & 65.8 & 0.6 & 125.5 & 1.2 & Vinegar, acid, fatty \\
\hline 73 & Propionic acid"* & 1573 & 1.1.0. & & n.d. & & 2.5 & 0.2 & 5.5 & 0.8 & 3.8 & 0.4 & n.d. & & n.d. & & n.d. & & n.d. & & Vinegarish \\
\hline 74 & Isobutyric acid" & 1601 & 14.8 & 0.2 & 11.5 & 0.5 & 9.3 & 0.5 & 46.4 & 2.0 & 45.2 & 4.0 & 41.9 & 1.1 & 19.0 & 3.5 & 19.4 & 0.6 & 19.9 & 0.4 & $\begin{array}{l}\text { Rancid, butter, } \\
\text { cheese }\end{array}$ \\
\hline 75 & Butyric acid" & 1664 & 10.2 & 0.2 & 9.0 & 0.1 & 8.4 & 0.2 & 22.0 & 0.5 & 21.1 & 0.5 & 19.6 & 0.7 & 8.8 & 0.1 & 8.2 & 0.2 & 9.1 & 0.1 & $\begin{array}{l}\text { Cheese, rancid, } \\
\text { sweat }\end{array}$ \\
\hline 76 & Isovaleric acid" & 1704 & n.d. & & n.d. & & n.d. & & 32.2 & 1.0 & n.d. & & n.d. & & 15.6 & 0.3 & 13.8 & 0.5 & 12.2 & 0.0 & Fatty, rancid \\
\hline 77 & 2-Methyl butyric acid"* & 1704 & 13.1 & 0.3 & n.d. & & n.d. & & 69.7 & 1.6 & 47.9 & 6.3 & n.d. & & 31.8 & 0.5 & 30.9 & 1.4 & 24.8 & 0.4 & Cheese, sweat \\
\hline 78 & Valeric acid" & 1773 & 0.9 & 0.1 & 0.8 & 0.1 & 1.1 & 0.0 & 1.9 & 0.1 & 1.5 & 0.1 & 1.6 & 0.1 & 1.3 & 0.0 & 1.5 & 0.1 & 1.5 & 0.0 & - \\
\hline 79 & Caproic acid" & 1880 & 92.9 & 3.4 & 88.2 & 1.0 & 103.5 & 4.1 & 308.61 & 6.65 & 301.32 & 12.31 & 294.95 & 3.78 & 152.3 & 2.3 & 140.7 & 3.2 & 148.2 & 2.1 & $\begin{array}{l}\text { Cheese, rancid, } \\
\text { fatty }\end{array}$ \\
\hline 80 & 2-Ethylhexanoic acid"* & 1981 & 13.0 & 1.3 & n.d. & & n.d. & & n.d. & & n.d. & & 17.8 & 2.4 & 19.5 & 1.8 & 11.5 & 1.6 & 0.0 & 0.0 & - \\
\hline 81 & (E)-4-Hexenoic acid ${ }^{* * * *}$ & 1996 & n.d. & & n.d. & & n.d. & & n.d. & & n.d. & & n.d. & & 13.0 & 1.1 & 8.5 & 1.4 & 12.4 & 1.7 & - \\
\hline 82 & (E)-2-Hexenoic acid" & 2003 & 28.8 & 1.7 & 33.6 & 0.2 & 36.6 & 1.2 & 16.2 & 1.4 & 18.5 & 1.1 & 17.8 & 0.7 & 15.0 & 0.8 & 10.0 & 1.5 & 21.6 & 0.8 & Musty, fatty \\
\hline 83 & Caprylic acid" & 2094 & 200.7 & 6.6 & 199.7 & 7.4 & 240.3 & 17.4 & 953.7 & 47.6 & 946.0 & 76.5 & 856.6 & 12.4 & 448.9 & 2.2 & 371.3 & 10.7 & 317.5 & 9.2 & $\begin{array}{l}\text { Rancid, harsh, } \\
\text { cheese, fatty acid }\end{array}$ \\
\hline 84 & Pelargonic acid & 2202 & n.d. & & n.d. & & n.d. & & n.d. & & n.d. & & n.d. & & 13.9 & 1.8 & n.d. & & n.d. & & Green, fatty \\
\hline 85 & Capric acid" & 2307 & 109.4 & 5.1 & 107.6 & 3.6 & 94.1 & 9.4 & 304.4 & 21.0 & 355.5 & 20.8 & 284.9 & 13.5 & 216.6 & 4.1 & 186.0 & 5.6 & 155.1 & 1.0 & Rancid, fatty \\
\hline 86 & Benzoic acid"* & 2482 & 30.8 & 3.7 & 26.7 & 5.0 & 36.2 & 2.4 & 25.2 & 1.2 & 33.0 & 4.7 & 40.6 & 2.8 & 45.3 & 1.1 & 37.3 & 7.0 & 29.5 & 3.2 & Urine \\
\hline 87 & Lauric acid ${ }^{* *}$ & 2519 & n.d. & & 26.4 & 2.5 & n.d. & & 53.1 & 9.4 & 40.8 & 3.5 & 60.4 & 9.0 & 44.9 & 2.2 & n.d. & & 58.8 & 4.4 & Metal \\
\hline 88 & Phenylacetic acid"* & 2608 & 12.1 & 0.5 & 12.8 & 0.1 & 13.9 & 0.6 & 31.5 & 2.5 & 32.0 & 2.0 & 28.5 & 2.0 & 32.3 & 0.9 & 33.2 & 1.9 & 32.3 & 1.1 & Honey, flower \\
\hline & Volatile phenols (19) & & & & & & & & & & & & & & & & & & & & \\
\hline 89 & Ethyl salicylate & 1839 & 17.4 & 0.3 & 10.0 & 0.2 & 9.0 & 0.8 & 6.7 & 0.4 & 6.2 & 0.6 & 7.8 & 0.6 & n.d. & & 29.9 & 1.0 & 5.1 & 0.4 & Wintergreen, mint \\
\hline 90 & Guaiacol $^{* *}$ & 1892 & 2.2 & 0.2 & 4.3 & 0.3 & 27.8 & 1.2 & n.d. & & n.d. & & 22.4 & 0.4 & n.d. & & n.d. & & n.d. & & $\begin{array}{l}\text { Smoky, sweet, } \\
\text { medicine }\end{array}$ \\
\hline 91 & 4-Ethylguaiacol ${ }^{*}$ & 2065 & n.d. & & n.d. & & n.d. & & 1.9 & 0.1 & 1.8 & 0.1 & 2.2 & 0.1 & 5.7 & 0.2 & 5.3 & 0.2 & 6.8 & 0.2 & Spice, clove \\
\hline 92 & $m$-Cresol ${ }^{* *}$ & 2120 & n.d. & & n.d. & & 7.3 & 1.3 & n.d. & & n.d. & & n.d. & & n.d. & & n.d. & & n.d. & & Leather \\
\hline
\end{tabular}




\begin{tabular}{|c|c|c|c|c|c|c|c|c|c|c|c|c|c|c|c|c|c|c|c|c|c|}
\hline 93 & 4-Ethylphenol ${ }^{-*}$ & 2215 & n.d. & & n.d. & & 6.0 & 0.9 & 8.6 & 0.4 & 6.7 & 0.8 & 9.0 & 0.9 & 8.5 & 1.4 & 10.1 & 0.6 & 11.1 & 1.0 & Phenolic \\
\hline 94 & 4-Vinylguaiacol ${ }^{*}$ & 2234 & n.d. & & 1.7 & 0.0 & 4.5 & 0.6 & n.d. & & 1.6 & 0.3 & 13.9 & 0.3 & n.d. & & n.d. & & 40.6 & 2.1 & Clove-like, smoky \\
\hline 95 & Syringol & 2301 & n.d. & & 16.1 & 2.1 & 107.2 & 7.6 & n.d. & & n.d. & & 118.6 & 17.4 & n.d. & & n.d. & & n.d. & & - \\
\hline 96 & 2,4-Di-tert-butylphenol ${ }^{* *}$ & 2346 & 25.9 & 1.4 & 31.1 & 1.8 & 35.8 & 2.1 & 43.9 & 6.3 & 38.8 & 2.8 & 40.0 & 5.2 & 50.7 & 3.9 & 36.4 & 1.7 & 49.9 & 1.4 & - \\
\hline 97 & 4-Vinylphenol ${ }^{*+*}$ & 2440 & n.d. & & n.d. & & n.d. & & n.d. & & n.d. & & 144.8 & 10.8 & n.d. & & n.d. & & 166.7 & 10.2 & Almond shell \\
\hline 98 & 4-Allylsyringol"* & 2579 & n.d. & & n.d. & & n.d. & & n.d. & & n.d. & & 18.8 & 0.2 & n.d. & & n.d. & & n.d. & & - \\
\hline 99 & 4-Ethoxymethylphenol ${ }^{* * * *}$ & 2586 & n.d. & & n.d. & & n.d. & & n.d. & & n.d. & & n.d. & & n.d. & & 14.0 & 2.4 & n.d. & & - \\
\hline 100 & Vanillin & 2613 & 38.4 & 1.0 & 42.5 & 0.8 & 39.5 & 1.4 & 13.7 & 0.9 & 27.1 & 0.7 & 17.0 & 0.9 & 22.2 & 0.5 & 54.4 & 2.2 & 28.2 & 1.3 & Vanilla-like, sweet \\
\hline 101 & Methyl vanillate ${ }^{* *}$ & 2650 & 9.9 & 1.4 & 11.2 & 1.3 & 16.2 & 1.8 & 20.0 & 2.6 & 16.6 & 1.6 & 20.1 & 2.6 & 12.3 & 1.5 & 12.8 & 2.5 & 23.3 & 3.6 & $\begin{array}{l}\text { Caramel, } \\
\text { butterscotch, } \\
\text { vanilla }\end{array}$ \\
\hline 102 & Ethyl vanillate $^{* * *}$ & 2676 & 47.7 & 2.8 & n.d. & & n.d. & & 51.7 & 4.3 & 96.0 & 6.5 & 86.7 & 5.0 & n.d. & & n.d. & & n.d. & & $\begin{array}{l}\text { Flowery, fruity, } \\
\text { sweet, vanilla }\end{array}$ \\
\hline 103 & Acetovanillone" & 2687 & 17.7 & 1.8 & 24.3 & 0.6 & 18.3 & 1.4 & 26.9 & 4.2 & 31.1 & 6.2 & 33.7 & 4.0 & 26.7 & 2.0 & 66.7 & 6.4 & 49.4 & 4.4 & Vanilla \\
\hline 104 & Vanillyl ethyl ether ${ }^{* * *}$ & 2780 & n.d. & & n.d. & & n.d. & & 30.7 & 3.7 & 32.8 & 4.4 & 65.7 & 12.9 & n.d. & & n.d. & & n.d. & & - \\
\hline 105 & 3,4-Dimethoxyphenol ${ }^{* * *}$ & 2816 & n.d. & & n.d. & & n.d. & & 14.8 & 2.3 & n.d. & & n.d. & & n.d. & & n.d. & & n.d. & & - \\
\hline 106 & Syringaldehyde & 2989 & 54.6 & 4.8 & 94.3 & 29.2 & 60.1 & 3.2 & n.d. & & 63.5 & 14.3 & 42.2 & 11.8 & n.d. & & 27.6 & 5.3 & n.d. & & $\begin{array}{l}\text { Chocolate, grape, } \\
\text { woody, smoky, } \\
\text { wine-like }\end{array}$ \\
\hline 107 & 4-Hydroxybenzaldehyde ${ }^{* * *}$ & 3015 & 101.8 & 11.1 & 105.0 & 7.8 & 104.2 & 10.0 & 51.3 & 7.6 & 177.1 & 15.9 & 107.9 & 12.0 & 139.5 & 2.0 & 268.8 & 21.7 & 46.5 & 5.2 & - \\
\hline 108 & $\begin{array}{l}\text { Monoterpenes (15) } \\
\text { Eucalyptol" }^{*}\end{array}$ & 1218 & n.d. & & n.d. & & n.d. & & n.d. & & n.d. & & n.d. & & n.d. & & n.d. & & 2.4 & 0.2 & $\begin{array}{l}\text { Eucalyptus, fresh, } \\
\text { cool, } \\
\text { camphoraceous, } \\
\text { medicinal }\end{array}$ \\
\hline 109 & $(E)$-Linalool oxide & 1462 & n.d. & & 6.5 & 0.5 & 19.7 & 0.6 & 4.0 & 0.2 & 10.7 & 0.7 & 26.8 & 0.2 & 4.4 & 0.6 & 13.1 & 0.4 & 31.7 & 1.2 & - \\
\hline 110 & (Z)-Linalool oxide" & 1490 & n.d. & & n.d. & & n.d. & & n.d. & & n.d. & & 10.0 & 0.3 & n.d. & & n.d. & & n.d. & & Flowery \\
\hline 111 & Linalool" & 1569 & n.d. & & n.d. & & n.d. & & n.d. & & n.d. & & n.d. & & 19.4 & 0.3 & n.d. & & n.d. & & $\begin{array}{l}\text { Flowery, citrus- } \\
\text { like, bergamot }\end{array}$ \\
\hline 112 & 3-Terpinen-1-ol ${ }^{* *}$ & 1597 & n.d. & & n.d. & & n.d. & & n.d. & & n.d. & & n.d. & & n.d. & & n.d. & & 2.2 & 0.3 & Musty \\
\hline 113 & Hotrienol $^{*}$ & 1634 & n.d. & & n.d. & & n.d. & & n.d. & & n.d. & & n.d. & & 3.3 & 0.3 & n.d. & & n.d. & & Hyacinth \\
\hline 114 & $\alpha$-Terpineol ${ }^{*}$ & 1720 & 3.7 & 0.4 & 8.0 & 0.7 & 8.0 & 1.3 & 5.9 & 0.4 & 8.0 & 0.3 & 8.0 & 0.7 & 42.4 & 0.5 & 39.9 & 1.2 & 18.1 & 1.8 & $\begin{array}{l}\text { Pine, oil, anise, } \\
\text { mint }\end{array}$ \\
\hline 115 & $\begin{array}{l}\text { 3,7-Dimethyl-1,5-octadien- } \\
\text { 3,7-diol }\end{array}$ & 1723 & n.d. & & n.d. & & n.d. & & n.d. & & n.d. & & n.d. & & 7.3 & 0.1 & n.d. & & n.d. & & - \\
\hline 116 & Citronellol $^{*}$ & 1788 & n.d. & & n.d. & & n.d. & & 3.9 & 0.3 & n.d. & & n.d. & & n.d. & & n.d. & & n.d. & & $\begin{array}{l}\text { Citronella, rose, } \\
\text { green, lemon }\end{array}$ \\
\hline 117 & Nerol $^{*}$ & 1825 & n.d. & & n.d. & & n.d. & & n.d. & & n.d. & & n.d. & & 4.1 & 0.5 & n.d. & & n.d. & & Sweet \\
\hline 118 & Geraniol $^{* *}$ & 1872 & n.d. & & n.d. & & n.d. & & n.d. & & n.d. & & n.d. & & 6.3 & 0.2 & n.d. & & n.d. & & $\begin{array}{l}\text { Rose-like, citrus- } \\
\text { like, geranium }\end{array}$ \\
\hline 119 & $\begin{array}{l}\text { 2,6-Dimethyl-3,7-octadien- } \\
\text { 2,6-diol }\end{array}$ & 1974 & n.d. & & n.d. & & n.d. & & n.d. & & n.d. & & n.d. & & 49.5 & 0.9 & 7.2 & 1.0 & n.d. & & - \\
\hline 120 & Linalool hydrate" & 2004 & n.d. & & n.d. & & n.d. & & n.d. & & n.d. & & n.d. & & 18.8 & 0.8 & 24.1 & 0.6 & n.d. & & - \\
\hline 121 & $p$-Menthane-3,8-diol ${ }^{* * *}$ & 2127 & n.d. & & n.d. & & n.d. & & n.d. & & 13.2 & 0.7 & 22.4 & 1.8 & n.d. & & n.d. & & n.d. & & - \\
\hline 122 & $\begin{array}{l}\text { 3,7-Dimethyl-1,7- } \\
\text { octanediol }^{*+}\end{array}$ & 2235 & n.d. & & n.d. & & n.d. & & 8.2 & 1.4 & n.d. & & n.d. & & 11.5 & 0.7 & n.d. & & n.d. & & - \\
\hline 123 & $\begin{array}{l}\text { Norisoprenoids (6) } \\
\text { 2,6,6-Trimethyl-2- } \\
\text { cyclohexen-1-one }\end{array}$ & 1422 & n.d. & & n.d. & & 2.8 & 0.2 & n.d. & & n.d. & & 3.7 & 0.6 & n.d. & & n.d. & & 2.6 & 0.4 & - \\
\hline 0.3 & Vitispirane & 1546 & n.d. & & 2.5 & & 2.9 & 0.3 & n.d. & & n.d. & & 4.7 & 0.5 & n.d. & & 13.8 & 0.7 & 11.5 & 0.4 & $\begin{array}{l}\text { Camphor, } \\
\text { eucalyptus }\end{array}$ \\
\hline & $\begin{array}{l}\text { 1-(2,4,6-Trimethylphenyl)buta- } \\
\text { 1,3-diene }\end{array}$ & 1768 & n.d. & & n.d. & & n.d. & & n.d. & & n.d. & & n.d. & & n.d. & & 1.4 & 0.1 & 1.8 & 0.1 & - \\
\hline 0.1 & $\begin{array}{l}\text { 1,1,6-Trimethyl-1,2- } \\
\text { dihydronaphthalene (TDN) }\end{array}$ & 1770 & n.d. & & 0.8 & & 1.2 & 0.1 & n.d. & & n.d. & & 1.9 & 0.1 & n.d. & & n.d. & & n.d. & & $\begin{array}{l}\text { Petrol, kerosene- } \\
\text { like }\end{array}$ \\
\hline 0.3 & $\beta$-Damascenone & 1846 & n.d. & & 3.5 & & 12.0 & 1.7 & n.d. & & n.d. & & n.d. & & n.d. & & n.d. & & 14.9 & 1.8 & $\begin{array}{l}\text { Baked apple, rose, } \\
\text { honey }\end{array}$ \\
\hline 128 & $\begin{array}{l}\text { 1-(2,3,6-Trimethylphenyl)-3- } \\
\text { buten-2-one }\end{array}$ & 2146 & n.d. & & n.d. & & 36.9 & 4.9 & n.d. & & n.d. & & 53.7 & 4.4 & n.d. & & n.d. & & 51.5 & 1.2 & - \\
\hline & Carbonyl compounds (18) & & & & & & & & & & & & & & & & & & & & \\
\hline 129 & 3-Hexanone & 1079 & 6.2 & 0.6 & 5.3 & 0.7 & 17.4 & 0.7 & n.d. & & n.d. & & n.d. & & n.d. & & 5.0 & 1.0 & 19.2 & 1.3 & Ether, grape \\
\hline 130 & Hexanal $^{* *}$ & 1098 & 6.2 & 0.4 & 5.3 & 0.6 & 4.8 & 0.5 & n.d. & & n.d. & & n.d. & & 5.6 & 0.5 & 4.7 & 0.8 & 5.8 & 0.9 & Grass, tallow, fatty \\
\hline 131 & (E)-3-Penten-2-one & 1145 & 4.9 & 0.3 & 3.7 & 0.5 & 4.7 & 0.8 & n.d. & & 8.7 & 0.8 & n.d. & & 5.5 & 0.7 & 5.4 & 0.2 & 5.3 & 0.7 & - \\
\hline
\end{tabular}




\begin{tabular}{|c|c|c|c|c|c|c|c|c|c|c|c|c|c|c|c|c|c|c|c|c|c|}
\hline \multirow[t]{2}{*}{ \# } & \multirow[t]{2}{*}{ Compounds } & \multirow[t]{2}{*}{$\mathrm{KI}$} & \multicolumn{6}{|c|}{ TN sweet $(\mu \mathrm{g} / \mathrm{L})$} & \multicolumn{6}{|c|}{ TN dry $(\mu \mathrm{g} / \mathrm{L})$} & \multicolumn{6}{|c|}{ Malvasia $(\mu \mathrm{g} / \mathrm{L})$} & \multirow{2}{*}{$\begin{array}{l}\text { Common } \\
\text { descriptors }^{a}\end{array}$} \\
\hline & & & $0 \mathrm{~m}$ & $\pm \mathrm{SD}$ & $\begin{array}{l}3 \mathrm{~m}, \\
45^{\circ} \mathrm{C}\end{array}$ & $\pm \mathrm{SD}$ & $\begin{array}{l}1 \mathrm{~m}, \\
70^{\circ} \mathrm{C}\end{array}$ & $\pm \mathrm{SD}$ & $0 \mathrm{~m}$ & $\pm \mathrm{SD}$ & $\begin{array}{l}3 \mathrm{~m}, \\
45^{\circ} \mathrm{C}\end{array}$ & $\pm \mathrm{SD}$ & $\begin{array}{l}1 \mathrm{~m}, \\
70^{\circ} \mathrm{C}\end{array}$ & $\pm \mathrm{SD}$ & $0 \mathrm{~m}$ & $\pm \mathrm{SD}$ & $\begin{array}{l}3 \mathrm{~m}, \\
45^{\circ} \mathrm{C} \\
\end{array}$ & $\pm \mathrm{SD}$ & $\begin{array}{l}1 \mathrm{~m}, \\
70^{\circ} \mathrm{C}\end{array}$ & $\pm \mathrm{SD}$ & \\
\hline 132 & 4-Ethoxy-2-pentanone ${ }^{* * * *}$ & 1258 & 2.2 & 0.1 & 3.1 & 0.1 & 2.1 & 0.1 & n.d. & & 4.8 & 0.2 & 0.9 & 0.0 & 3.1 & 0.0 & 7.7 & 0.1 & 3.3 & 0.1 & - \\
\hline 133 & 4-ethoxy-2-butanone ${ }^{*+* * *}$ & 1280 & n.d. & & n.d. & & 4.5 & 0.3 & n.d. & & n.d. & & n.d. & & n.d. & & n.d. & & 3.6 & 0.1 & - \\
\hline 134 & Acetoin & 1309 & 20.0 & 0.2 & 20.1 & 0.4 & 19.9 & 0.4 & 11.9 & 0.2 & 12.0 & 0.6 & 10.1 & 0.0 & n.d. & & n.d. & & 38.8 & 1.0 & $\begin{array}{l}\text { Butter, cream, } \\
\text { flowery, wet }\end{array}$ \\
\hline 135 & Acetol $^{*+}$ & 1326 & n.d. & & 5.0 & 0.2 & 52.8 & 1.8 & n.d. & & n.d. & & n.d. & & n.d. & & n.d. & & 55.2 & 2.2 & Sweet, caramel \\
\hline 136 & Cyclopenten-3-one ${ }^{* \cdots}$ & 1379 & n.d. & & n.d. & & n.d. & & n.d. & & n.d. & & n.d. & & n.d. & & n.d. & & 3.6 & 0.6 & - \\
\hline 137 & Benzaldehyde & 1551 & 127.6 & 1.5 & 102.9 & 1.3 & 103.0 & 2.6 & 10.2 & 0.2 & 50.6 & 2.9 & 22.0 & 2.2 & 71.8 & 0.9 & 77.2 & 1.1 & 62.5 & 1.6 & $\begin{array}{l}\text { Almond, burnt } \\
\text { sugar }\end{array}$ \\
\hline 138 & 2-Cyclopentene-1,4-dione & 1616 & n.d. & & n.d. & & 7.1 & 0.5 & n.d. & & n.d. & & n.d. & & n.d. & & n.d. & & 9.8 & 0.7 & - \\
\hline 139 & Phenylacetaldehyde" & 1673 & 79.2 & 0.5 & 54.6 & 1.3 & 98.2 & 2.1 & 12.5 & 1.2 & 28.3 & 0.8 & 46.6 & 2.0 & 28.7 & 1.6 & 38.8 & 2.6 & 31.4 & 2.0 & Green, honey \\
\hline 140 & Phenylacetone ${ }^{* \ldots t}$ & 1756 & n.d. & & n.d. & & n.d. & & n.d. & & n.d. & & n.d. & & n.d. & & 6.0 & 0.2 & 3.7 & 0.1 & - \\
\hline 141 & Ethyl nicotinate ${ }^{* *}$ & 1843 & n.d. & & 3.5 & 0.1 & 7.6 & 0.3 & n.d. & & n.d. & & 10.0 & 0.2 & n.d. & & n.d. & & 6.9 & 0.1 & - \\
\hline 142 & Cyclotene $^{* * * *}$ & 1859 & n.d. & & n.d. & & 9.6 & 1.2 & n.d. & & n.d. & & n.d. & & n.d. & & n.d. & & n.d. & & Sweet, burnt \\
\hline 143 & Ethyl picolinate $^{* \ldots * *}$ & 2028 & n.d. & & n.d. & & 13.8 & 0.5 & n.d. & & n.d. & & n.d. & & n.d. & & n.d. & & n.d. & & - \\
\hline 144 & 2H-Pyran-2,6(3H)-dione ${ }^{* * *}$ & 2033 & n.d. & & n.d. & & n.d. & & 11.1 & 0.7 & 5.8 & 0.8 & 18.1 & 1.5 & n.d. & & n.d. & & n.d. & & \\
\hline 145 & 3-Hydroxy-4-phenyl-2-butanone & 2302 & n.d. & & n.d. & & n.d. & & n.d. & & n.d. & & n.d. & & 20.6 & 1.7 & 27.0 & 5.7 & 25.7 & 1.2 & - \\
\hline \multirow[t]{2}{*}{146} & Hydroxymaltol ${ }^{* * *}$ & 2332 & n.d. & & 49.8 & 4.8 & 81.9 & 10.7 & n.d. & & n.d. & & n.d. & & n.d. & & 119.4 & 8.8 & 31.7 & 3.3 & - \\
\hline & Furan compounds (15) & & & & & & & & & & & & & & & & & & & & \\
\hline 147 & $\begin{array}{l}\text { 2,2-Dimethyl-5-(1-methyl-1- } \\
\text { propenyl)-tetrahydrofuran }\end{array}$ & 1258 & n.d. & & n.d. & & n.d. & & n.d. & & n.d. & & n.d. & & n.d. & & n.d. & & 3.0 & 0.1 & - \\
\hline 148 & $\begin{array}{l}\text { Dihydro-2-methyl-3(2H)- } \\
\text { furanone }\end{array}$ & 1286 & n.d. & & n.d. & & 8.5 & 0.3 & n.d. & & n.d. & & n.d. & & n.d. & & n.d. & & 9.7 & 0.2 & - \\
\hline 149 & Furfural $^{*}$ & 1492 & 8.0 & 0.1 & 159.4 & 3.3 & 987.5 & 16.1 & 5.6 & 0.0 & 98.8 & 1.6 & 563.1 & 18.0 & 9.2 & 0.2 & 143.9 & 2.2 & 1038.2 & 14.4 & $\begin{array}{l}\text { Almonds, pungent, } \\
\text { bread, sweet }\end{array}$ \\
\hline 150 & 2-Acetylfuran" & 1533 & n.d. & & 3.3 & 0.0 & 29.1 & 0.9 & n.d. & & 2.3 & 0.1 & 15.8 & 0.4 & n.d. & & 3.5 & 0.0 & 26.3 & 0.4 & Balsamic \\
\hline 151 & 5-Methylfurfural ${ }^{*}$ & 1600 & n.d. & & 6.9 & 0.1 & 116.7 & 3.3 & n.d. & & 4.2 & 0.1 & 50.1 & 1.7 & n.d. & & 5.2 & 0.1 & 98.1 & 2.8 & $\begin{array}{l}\text { Almond, caramel, } \\
\text { burnt sugar }\end{array}$ \\
\hline 152 & 2-Furyl ethyl ketone & 1602 & n.d. & & n.d. & & 16.3 & 0.4 & n.d. & & n.d. & & n.d. & & n.d. & & n.d. & & 25.9 & 0.3 & - \\
\hline 153 & 2-Acetyl-5-methylfuran & 1642 & n.d. & & n.d. & & 6.7 & 0.4 & n.d. & & n.d. & & 6.8 & 1.6 & n.d. & & n.d. & & 10.8 & 0.6 & - \\
\hline 154 & Ethyl 2 -furoate & 1650 & 5.8 & 0.8 & 12.7 & 0.4 & 44.1 & 0.8 & 5.5 & 1.0 & 12.0 & 0.5 & 57.9 & 1.4 & 12.3 & 0.6 & 21.6 & 1.5 & 76.9 & 1.6 & Vanilla, scorched \\
\hline 155 & Furfuryl alcohol ${ }^{* *}$ & 1690 & n.d. & & 2.6 & 0.4 & 9.7 & 0.6 & 4.9 & 0.9 & n.d. & & n.d. & & n.d. & & n.d. & & 13.5 & 2.0 & Burnt \\
\hline 156 & 5-Ethoxymethylfurfural ${ }^{l * * *}$ & 1962 & n.d. & & 52.3 & 1.5 & 3616.4 & 114.8 & n.d. & & n.d. & & 117.7 & 9.8 & n.d. & & 26.2 & 1.7 & 3551.3 & 33.1 & - \\
\hline 157 & 2,5-Furandicarboxaldehyde ${ }^{* * *}$ & 2018 & n.d. & & 6.6 & 0.4 & 101.5 & 7.4 & n.d. & & n.d. & & n.d. & & n.d. & & n.d. & & 128.8 & 9.7 & - \\
\hline 158 & Furyl hydroxymethyl ketone ${ }^{* * *}$ & 2046 & 1.3 & 0.0 & 29.0 & 0.3 & 355.4 & 13.4 & n.d. & & n.d. & & 9.4 & 0.3 & 1.3 & 0.0 & 18.6 & 0.4 & 458.5 & 9.2 & - \\
\hline 159 & 2,3-Dihydrobenzofuran ${ }^{* * * *}$ & 2437 & n.d. & & 21.2 & 1.4 & 44.2 & 4.2 & n.d. & & n.d. & & n.d. & & n.d. & & n.d. & & n.d. & & - \\
\hline 160 & 5-Hydroxymethylfurfural (HMF) & 2551 & 41.3 & 0.8 & 3124.7 & 141.3 & 20738.1 & 1533.3 & 35.7 & 14.8 & 82.0 & 5.4 & 868.7 & 49.7 & 32.0 & 2.3 & 1518.1 & 97.7 & 19571.7 & 284.4 & Caramel \\
\hline 161 & $\begin{array}{l}\text { 1-(5-Hydroxymethyl-2-furanyl)-1- } \\
\text { propanone }\end{array}$ & 2623 & n.d. & & n.d. & & 37.0 & 1.9 & n.d. & & n.d. & & n.d. & & n.d. & & n.d. & & 131.7 & 6.7 & - \\
\hline & Lactones (7) & & & & & & & & & & & & & & & & & & & & \\
\hline 162 & $\gamma$-Butyrolactone & 1661 & 16.1 & 0.2 & 17.4 & 0.6 & 17.0 & 0.4 & 40.6 & 0.8 & 44.1 & 0.6 & 44.3 & 1.6 & 13.0 & 0.3 & 13.1 & 0.1 & 14.3 & 0.3 & Caramel, sweet \\
\hline 163 & $\alpha$-Angelica lactone ${ }^{\omega * *}$ & 1460 & n.d. & & n.d. & & 3.9 & 0.4 & n.d. & & n.d. & & n.d. & & n.d. & & n.d. & & 7.9 & 1.2 & - \\
\hline 164 & $\beta$-Angelica lactone ${ }^{* * *}$ & 1709 & n.d. & & n.d. & & 6.1 & 0.1 & n.d. & & n.d. & & n.d. & & n.d. & & n.d. & & 6.9 & 0.2 & - \\
\hline 165 & $\gamma$-Ethoxybutyrolactone ${ }^{* * * *}$ & 1756 & 14.4 & 0.2 & 13.2 & 0.2 & 17.5 & 0.2 & 9.7 & 0.5 & 14.9 & 0.4 & 13.2 & 0.6 & 5.2 & 0.1 & 9.4 & 0.7 & 11.4 & 0.3 & - \\
\hline 166 & $\gamma$-Nonalactone & 2060 & n.d. & & 9.8 & 1.3 & n.d. & & 8.7 & 2.2 & 10.1 & 0.5 & 20.0 & 2.0 & n.d. & & 7.1 & 0.7 & 9.0 & 1.7 & Coconut, peach \\
\hline 167 & $\gamma$-Decalactone ${ }^{* * *}$ & 2112 & n.d. & & n.d. & & 2.6 & 0.1 & 2.9 & 0.1 & 3.4 & 0.3 & 8.4 & 0.5 & n.d. & & n.d. & & n.d. & & $\begin{array}{l}\text { Peach, fatty, } \\
\text { coconut }\end{array}$ \\
\hline \multirow[t]{2}{*}{168} & $\begin{array}{l}\gamma \text {-Carboethoxy- } \gamma- \\
\text { butyrolactone }\end{array}$ & 2273 & 79.9 & 1.0 & 118.4 & 1.8 & 125.0 & 3.8 & 364.9 & 16.7 & 467.9 & 9.3 & 539.3 & 23.5 & 140.4 & 3.9 & 190.5 & 2.8 & 235.3 & 10.4 & Roast, smoke \\
\hline & Sulphur compounds (4) & & & & & & & & & & & & & & & & & & & & \\
\hline 169 & 2-Methyl-3-thiolanone ${ }^{* * * *}$ & 1556 & n.d. & & n.d. & & n.d. & & 8.4 & 0.2 & 6.3 & 0.5 & 12.5 & 1.1 & n.d. & & n.d. & & 4.9 & 0.6 & - \\
\hline 170 & $\begin{array}{l}\text { Ethyl 3-(methylthio) } \\
\text { propionate }\end{array}$ & 1591 & n.d. & & n.d. & & n.d. & & 11.3 & 0.7 & 14.2 & 2.0 & 17.4 & 2.2 & n.d. & & 3.1 & 0.4 & 3.4 & 0.3 & - \\
\hline 171 & Methionol $^{*}$ & 1747 & n.d. & & n.d. & & n.d. & & 161.9 & 4.0 & 135.4 & 4.9 & 137.9 & 3.3 & 10.5 & 0.4 & 9.4 & 1.3 & 10.1 & 0.7 & Sweet, potato \\
\hline 172 & 4-(Methylthio)-1-butanol ${ }^{\cdots *}$ & 1870 & n.d. & & n.d. & & n.d. & & 9.3 & 0.4 & 7.2 & 0.8 & 8.5 & 0.9 & n.d. & & n.d. & & n.d. & & - \\
\hline 173 & $\begin{array}{l}\text { Acetals (8) } \\
\text { Isovaleraldehyde diethyl }\end{array}$ & 1090 & n.d. & & n.d. & & 20.4 & 0.7 & n.d. & & n.d. & & 10.9 & 3.3 & n.d. & & n.d. & & 7.0 & 0.5 & - \\
\hline
\end{tabular}




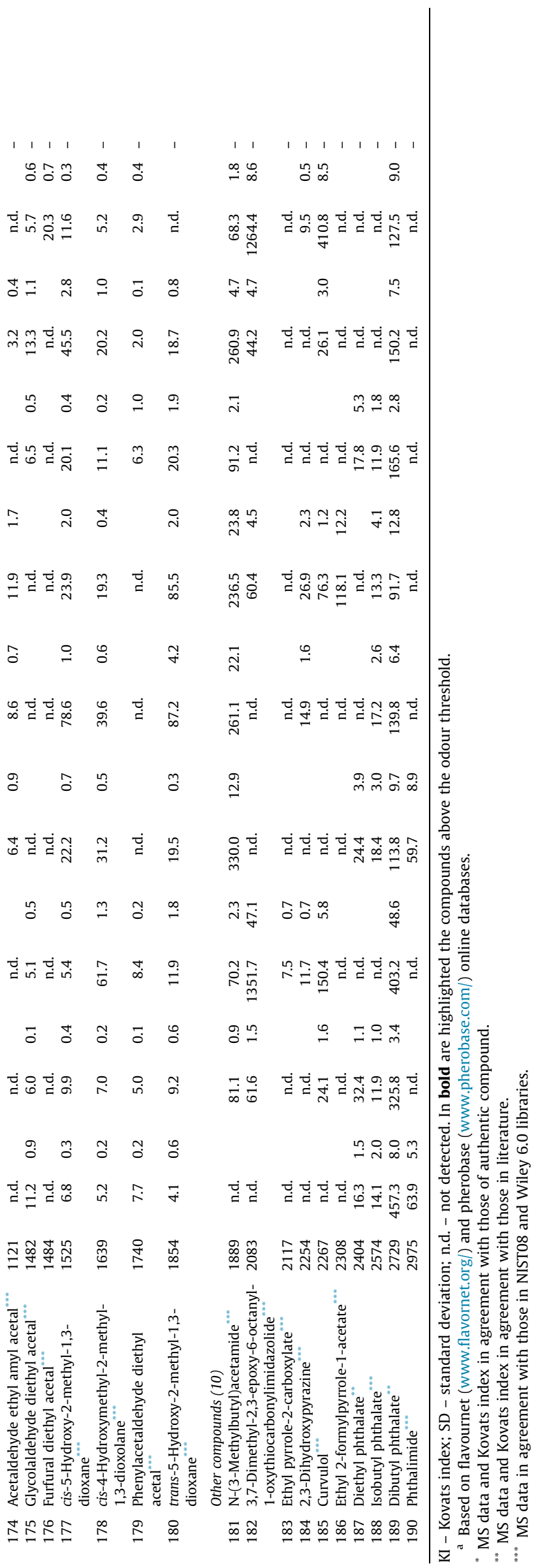

(Fig. 3). This study also showed that the volatile fraction of these Madeira wines increased after the heating process, up to $88 \%$ in sweet wines and up to $28 \%$ in the dry wine.

\subsection{Esters}

Esters are usually considered important to the sensory properties of wines, contributing with positive aromas, essentially with fruity notes (Ebeler, 2001). The results showed that esters were one of the most abundant groups (representing at least 30\%) as they are secondary aromas (Fig. 3). As expected, they appeared at higher amounts in the dry wine because it was more fermented.

Most esters increased with the heating step, mainly the most abundant (Table 1), which were diethyl malate (odour threshold (Oth) of $760 \mathrm{mg} / \mathrm{L}$, (Sánchez-Palomo, Gómez García-Carpintero, Alonso-Villegas, \& González-Viñas, 2010)), ethyl hydrogen succinate, diethyl succinate and ethyl lactate (Oth of 200 and $155 \mathrm{mg} /$ L, respectively (Campo, Ferreira, Escudero, Marques, \& Cacho, 2006), but none seem to contribute to the flavour of the current wines, because they were below their Oth. Other minor esters also increased (ethyl butyrate and ethyl caproate) or were formed (ethyl 2-methylbutyrate and ethyl isovalerate) after the heating step. These ethyl esters might impart positive fruity notes to the aroma of these wines since they were above their Oth $(20,18,3$ and $14 \mu \mathrm{g} / \mathrm{L}$, respectively; Campo et al., 2006; Gómez-Míguez, Cacho, Ferreira, Vicario, \& Heredia, 2007). Ethyl caproate has been reported as an important odorant of young TN wines (Perestrelo, Fernandes, Albuquerque, Marques, \& Câmara, 2006), as well as of aged Madeira wines (Campo et al., 2006).

Other minor esters revealed a great increase with baking. Some might even be important for the aroma definition of older Madeira wines, such as diethyl 2-hydroxyglutarate that was found to increase up to $125 \%$ after estufagem. Lee and Noble (2003) found that this ester contributes a cotton candy aroma to Californian Chardonnay wines. This compound has also been found in wines made from botrytised berries (Accordini, 2013). Another ester that showed a marked development was diethyl tartrate, which increased to almost 30-fold its initial value. On the other hand, some esters decreased after thermal processing, e.g., isoamyl acetate, which was present at levels higher than its Oth $(30 \mu \mathrm{g} / \mathrm{L}$, (Campo et al., 2006)) in the young TN dry wine, nevertheless its banana-like scent diminished due its decline.

There are several esters that only appeared after baking, namely the previously mentioned ethyls 2-methylbutyrate and isovalerate, but also ethyl levulinate, ethyl 3-ethoxypropionate, propyl ethyl hydroxypentanedioate, diethyl methylsuccinate and ethyl citrate. Ethyl levulinate was also encountered in sweet fortified wines by Cutzach et al. (1999), at forced-ageing conditions (heating at $37^{\circ} \mathrm{C}$ for 12 months). According to them, levulinic acid, which can be formed by the heat breakdown of glucose, furfuryl alcohol or HMF in acidic medium, reacts with ethanol, to form ethyl levulinate. Ethyl 3-ethoxypropionate, as far as we know, has only been identified in brandies (Ledauphin et al., 2004), while propyl ethyl hydroxypentanedioate has not been identified in beverages. Diethyl methylsuccinate was previously identified in oak-aged Madeira wines by A. C. Pereira, Reis, Saraiva, and Marques (2010). Actually, these authors, according to chemometric studies, found out that this ester, together with ethyl lactate, ethyl methylsuccinate, diethyl succinate and ethyl hydrogen succinate, were especially important in the aged wines. Ethyl citrate was also found by Schneider, Baumes, Bayonove, and Razungles (1998) in sweet fortified wines from Grenache Noir.

Some esters are not commonly reported in wines, namely ethyl phenylacetate and ethyl 4-hydroxyphenylacetate, which indeed increased after estufagem, however it has been found in Aglianico del Vulture wines by Tat, Comuzzo, Battistutta, and Zironi (2007) 


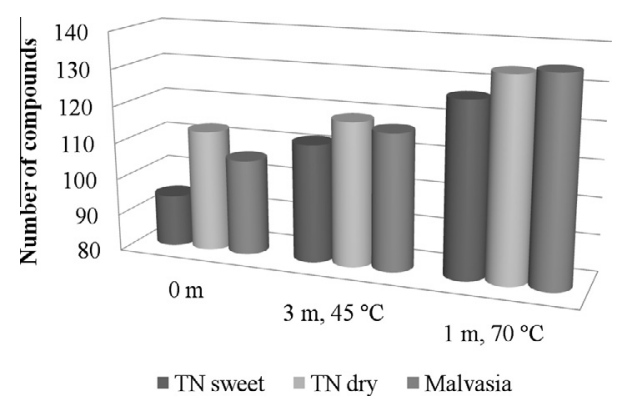

Fig. 2. Number of compounds identified before and after the heating at standard ( 3 months, $45^{\circ} \mathrm{C}$ ) and overheating $\left(1\right.$ month, $70^{\circ} \mathrm{C}$ ) conditions of the Madeira wines in study.

and in Riesling wines by Güntert, Rapp, Takeoka, and Jennings (1986). Ethyl phenylacetate occurrence might be related with shikimate (phenylalanine and tyrosine) and cinnamate pathways during alcoholic fermentation by enzymatic esterification of phenylacetic acid (Tat et al., 2007). Likewise, the occurrence of 4hydroxyphenylacetate might be related with the esterification of 4-hydroxyphenylacetic acid, a product of tyrosine metabolism (Güntert et al., 1986). Despite its positive development, the concentration of ethyl phenylacetate never attained its odour threshold, of $73 \mu \mathrm{g} / \mathrm{L}$ (Tat et al., 2007).

\subsection{Higher alcohols}

Higher alcohols were quantitatively the largest group of volatiles accumulated during the fermentation of these Madeira wines, representing in average $57 \%$ of the total content (Fig. 3). However, taking into account that they generally have high perception threshold, they did not seem to influence the aroma of these fortified wines.

These secondary aromas are essentially formed either from sugar catabolism or from amino acid decarboxylation and deamination. In this sense, unsurprisingly, sweet wines (short fermentation) presented the lowest content of alcohols (Fig. 3). From the 29 alcohols identified, active amyl alcohol, phenylethyl alcohol and 1hexanol were by far the most abundant higher alcohols, with concentrations between 1.0 and $16.5 \mathrm{mg} / \mathrm{L}$ (Table 1 ). After the thermal processing of these wines, alcohols did not change very much, only small variations were encountered, thus it was not possible to identify a clear tendency. Actually, only 2-ethoxybenzyl alcohol was formed during the heating step. As far as we know, this compound is rarely reported in wines and may be derived from the reaction of ethanol with benzyl alcohol, which indeed slightly decreased after estufagem (Table 1).

\subsection{Fatty acids}

Fatty acids in wines may have origin in grapes or can be formed during the fermentative step by microbial organisms. Several compounds belonging to this chemical family were identified in these wines, including short chain (propionic, butyric and valeric acids), medium chain (caproic, caprylic, pelargonic and capric acids), long chain (lauric acid) and branched-chain fatty acids (isobutyric, isovaleric, 2-methylbutyric, 2-ethylhexanoic, 4-hexenoic and (E)2-hexenoic acids). Together, they did not represent more than $3.5 \%$ of the volatile fraction of these wines (Fig. 3). Fatty acids were more abundant in TN dry wine (Table 1 ) due to the longer fermentation time.

The most abundant was caprylic acid, with levels ranging from 199.7 to $953.7 \mu \mathrm{g} / \mathrm{L}$. Actually it was the only fatty acid exceeding its odour threshold $(500 \mu \mathrm{g} / \mathrm{L})$, but only in TN dry wine. Uncom-
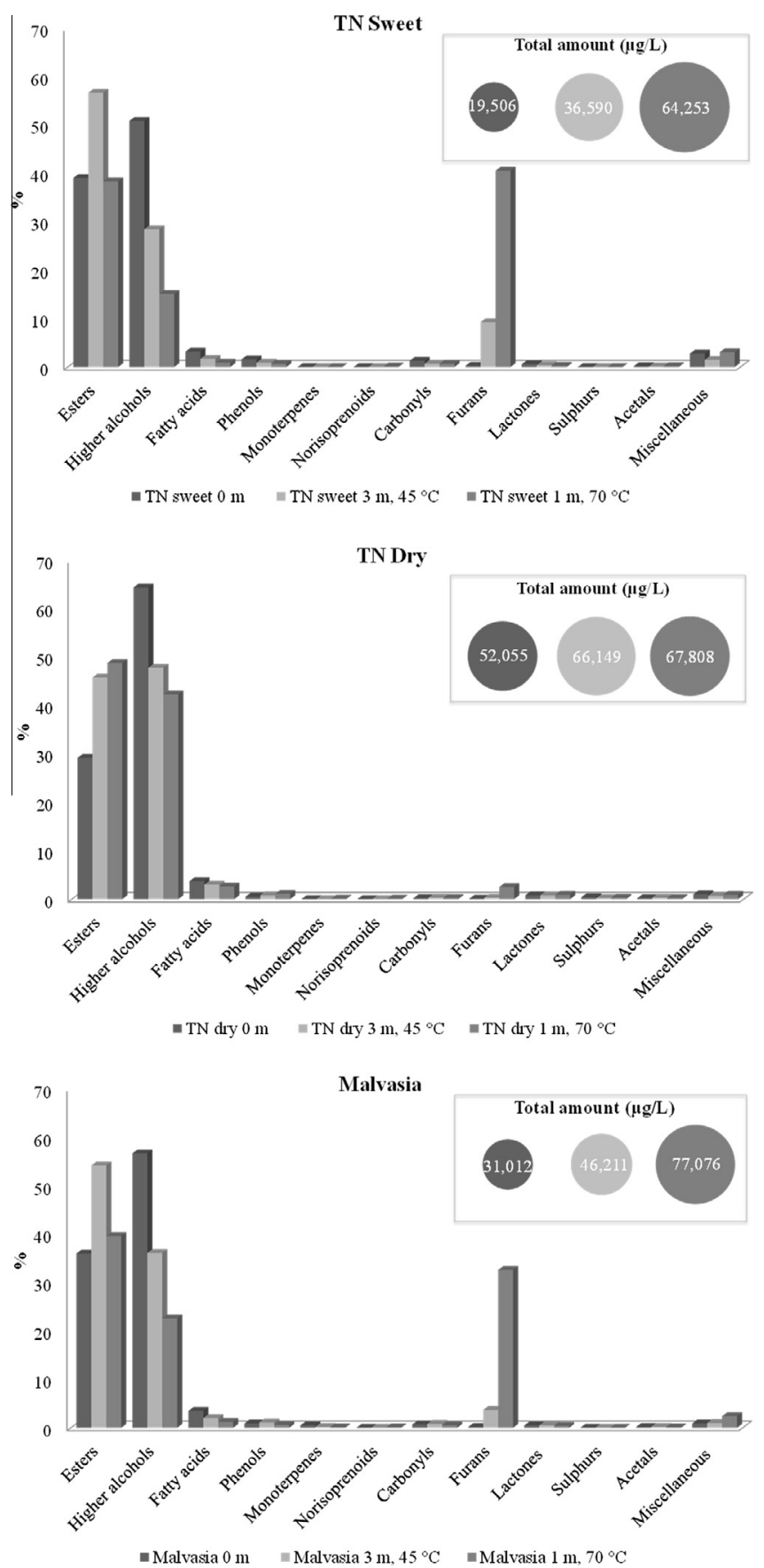

Fig. 3. Percentage of each chemical family identified in the current Madeira wines before and after heating at standard $\left(3\right.$ months at $\left.45^{\circ} \mathrm{C}\right)$ and overheating ( 1 month at $70^{\circ} \mathrm{C}$ ) conditions.

monly, 4-hexenoic acid was encountered in Malvasia wine. According to deMan (1999), the presence of this acid in wines is usually related with the microbial degradation of sorbate, generating a geranium off-flavour note. In general, fatty acids decreased after the baking step (Fig. 3), probably due to their participation in esterification reactions with ethanol.

\subsection{Volatile phenols}

Nineteen volatile phenols were identified in this sample set of Madeira wines. Quantitatively, they represent a minor group (less than $1.1 \%$ ) among the volatiles encountered. However, some of them may negatively affect the overall aroma of a wine, if present 
at concentrations above their low odour thresholds, imparting animal, horse sweat, leather or medicinal off-flavours. The most common examples are vinylphenols and ethylphenols. Actually, 4ethylphenol (Oth of $440 \mu \mathrm{g} / \mathrm{L}$, (Campo et al., 2006)), 4-ethylguaiacol (Oth of $33 \mu \mathrm{g} / \mathrm{L}$, (Gómez-Míguez et al., 2007)), 4-vinylphenol and 4-vinylguaiacol (Oth of 180 and $1100 \mu \mathrm{g} / \mathrm{L}$, respectively (Campo et al., 2006)) were identified in these wines but all bellow their odour threshold (Table 1), so it seems that they might not have an impact on the aroma of these wines. Guaiacol was found above its odour threshold (up to 3-fold higher) only in TN wines heated at $70{ }^{\circ} \mathrm{C}$. Guaiacol is frequently considered as a cause of defects in wines, imparting smoky notes. This result suggests that temperature accelerates its development; hence high temperatures should be avoided in the thermal processing. In wine, guaiacol occurrence is normally associated with oak barrel maturation, however, this route does not explain its development with the current heating. Probably, its development might be related with the thermal decarboxylation of hydroxycinnamates. Vanillin and its esters, acetovanillone, syringaldehyde and 4-hydroxybenzadehyde were also detected, but all bellow their odour perception limit. The occurrence of vanillin in wines is also commonly related with barrel maturation, but in this case this observation is not likely because these wines did not pass through wood-ageing yet. A reasonable explanation is that maybe vanillin and its esters were transferred from grapes to wines (Flamini \& Traldi, 2010). The slight increase of acetovanillone after wines heating is also interesting. Similarly, Escudero et al. (2000) also found acetovanillone in laboratory oxidised white wines. According to them, wine oxidation may release the glycosylated acetovanillone extracted from grapes. Others volatile phenols were only detected after baking, namely 4-vinylguaiacol, 4-vinylphenol, 4-allylsyringol and syringol. Generally speaking, volatile phenols seem to be promoted by the thermal treatment of wines.

\subsection{Monoterpenes}

Monoterpenic compounds are usually considered as varietal compounds because they are present in grapes, especially in skins, or arise from grape precursors. In the current sample set were found 15 monoterpenes, representing less than $0.5 \%$ of the volatile fraction. The highest levels were found in young Malvasia wine (white variety), with at least 8 -fold more.

In general, monoterpenic alcohols such as linalool, hotrienol, citronellol, nerol and geraniol, when present, disappeared with heating. It was also observed the same tendency for most monoterpenic diols, excepting $p$-menthane-3,8-diol, which was only detected in baked TN dry wine. It is known that the profile and content of monoterpenes can be altered during ageing, essentially due to acid-catalysed reactions. For example, linalool can be transformed into $\alpha$-terpineol and successively to 1,8 -terpines, and geraniol and nerol into linalool and $\alpha$-terpineol (Versini, Dellacassa, Carlin, Fedrizzi, \& Magno, 2008). Probably, this explains the arising of eucalyptol (1,8-cineole), 3-terpinen-1-ol, p-menthane-3,8-diol and the increasing of $\alpha$-terpineol in TN wines. According to Marais (1983) review, eucalyptol was also found in wines heated at $70^{\circ} \mathrm{C}$ (wine $\mathrm{pH}$ at 1.0 ). Monoterpenic oxides were also identified, namely the isomeric forms of linalool oxides, that abruptly increased after the heating process, while most monoterpenic diols tend to disappear after baking, like most monoterpenic alcohols. Linalool oxides may have been formed by linalool oxidation via epoxide (Marais, 1983).

\subsection{Norisoprenoids}

Six norisoprenoids were identified in these Madeira wines: 2,6,6trimethyl-2-cyclohexen-1-one, vitispirane, 1-(2,4,6-trimethyl- phenyl)buta-1,3-diene, TDN, $\beta$-damascenone and 1-(2,3,6-trimethylphenyl)-3-buten-2-one. These compounds were only detected in baked Madeira wines, indicating that temperature accelerates their formation. Kanasawud and Crouzet (1990) demonstrated that 2,6,6trimethyl-2-cyclohexen-1-one formation in aqueous medium is related with the thermal degradation of carotenoids, namely $\beta$-carotene. Apparently, this ketone has never been identified in wines.

In the current study, an isomer of TPB, the 1-(2,4,6-trimethylphenyl)buta-1,3-diene, was identified according to Wiley 6.0 MS library, with the following ion peaks $(\mathrm{m} / \mathrm{z})$, ordered according to their abundance: $157,142,141,128,172,115$, and eluting before $\beta$-damascenone with a KI of 1768. Janusz et al. (2003) were the first researchers to report the occurrence of TPB in wines as a potent grape-derived odorant, with a very low odour perception limit of $40 \mathrm{ng} / \mathrm{L}$. According to them, this compound exhibits green and cut-grass notes at low concentrations and pungent or chemical scents when present at higher concentrations. They reported that TPB elutes just after the elution of $\beta$-damascenone on a Carbowax column, with a Kovats GC retention index of 1830 and a mass spectrum with the following ions, ordered according to their abundance: $m / z 157,142,141,172,128,115$. Additionally, in the current study we also found another compound with structural similarities with TPB, the 1-(2,3,6-trimethylphenyl)-3-buten-2one, which, as far as we know, was only identified by Nykanen (1986) in wine and distilled alcoholic beverages.

Finally, vitispirane and TDN were never been encountered above their odour perception; however, $\beta$-damascenone, only detected in baked sweet wines, reaching 300 -fold above its odour threshold $(0.05 \mu \mathrm{g} / \mathrm{L}$, (Campo et al., 2006)) in wines heated at overheating conditions. This result leads us to conclude that this compound might play an important role in the aroma of Madeira baked wines. Campo et al. (2006) found that $\beta$-damascenone has a great contribution to the aroma of oak-aged Madeira wines.

\subsection{Carbonyl compounds}

Carbonyls group includes aldehydes and ketones. In the current sample set 18 carbonyls were found, from which 3 were aldehydes.

It is known that the major wine aldehyde is acetaldehyde; however, in the current samples it was not possible to quantify this aldehyde elutes too early to measure. However, other aldehydes were identified, namely hexanal, benzaldehyde and phenylacetaldehyde. The latter compound has reached values far above its odour perception (98-fold higher) and it seems that baking favours its development (Table 1). These results indicate that phenylacetaldehyde (honey-like scent) might be important for the aroma definition of the Madeira wines submitted to estufagem. Actually, Oliveira e Silva et al. (2008), through GC-O analysis, also found out that phenylacetaldehyde imparted a significant contribution to the aroma of heated Madeira wines. Comparable results were obtained by Campo et al. (2006) in oak-aged Madeira wines. The occurrence of this aldehyde in beverages has been related with Maillard reactions, specifically with Strecker degradation, through phenylalanine degradation (Soares da Costa et al., 2004). According to Campo et al. (2006) the presence of hexanal is most likely due to the direct oxidation of 1-hexanol.

Several ketones were identified in these wines, specifically some usually found in sweet fortified wines, namely cyclotene, 3-hydroxy-4-phenyl-2-butanone and hydroxymaltol (Cutzach et al., 1999; López de Lerma et al., 2010). Cyclotene and hydroxymaltol were only detected in baked wines at levels ranging from 9.6 to $119.4 \mu \mathrm{g} / \mathrm{L}$. The presence of these two carbonyls in wines is usually associated with Maillard reactions (Cutzach et al., 1999). Other carbonyls that are rarely identified in wines were also identified, namely (E)-3-penten-2-one, 4-ethoxy-2-pentanone, 4-ethoxy-2-butanone, cyclopenten-3-one, 2-cyclopentene-1, 
4-dione, phenylacetone, ethyl nicotinate, ethyl picolinate, and $2 \mathrm{H}$ pyran-2,6(3H)-dione. (E)-3-Penten-2-one was already encountered in young Madeiras by Perestrelo et al. (2006). Cyclopenten-3-one, only detected in Malvasia heated at $70^{\circ} \mathrm{C}$, has been detected in foods submitted to thermal processing, especially in coffee (Nebesny, Budryn, Kula, \& Majda, 2007). The formation of 2-cyclopentene-1,4-dione in foods has been related with sugar degradation promoted by heating, namely in Maillard reaction (Ames, Guy, \& Kipping, 2001). In fact, this compound was only found in the sweet wines submitted to heating. As far as we know, this carbonyl has never been detected in wines. It seems that phenylacetone formation is also associated with the heating process since it was only detected in baked Malvasia wines. Actually, it was identified in roasted model reactions consisting of glucose and phenylalanine (Baltes \& Mevissen, 1988). 2H-Pyran-2,6(3H)-dione was previously identified in oak aged Madeira wines (A. C. Pereira et al., 2010). It seems that ethyl nicotinate and picolinate were only detected in these baked wines.

\subsection{Furan compounds}

At least 15 furan compounds were developed in Madeira wines due to the thermal processing. Generally speaking, heating developed more furans in sweet wines than in dry wines as well as higher contents (Fig. 3). In sweet wines baked at standard conditions furans composed, on average, $7 \%$ of the volatile fraction, while in the dry wine they represent less than $0.3 \%$. Nevertheless, after heating at $70{ }^{\circ} \mathrm{C}$, furans comprised up to $41 \%$ in sweet wines and less than $3 \%$ in TN dry wine. These results can be explained by the fact that furans in wines are generated by the thermal degradation of sugars due to acid-catalysed reactions, or even through Maillard reaction (V. Pereira, Albuquerque, Ferreira, Cacho, \& Marques, 2011). Some furans provided remarkable increases with baking, especially 5hydroxymethylfurfural (HMF), 5-ethoxymethylfurfural, furfural, furyl hydroxymethyl ketone, 2,5-furandicarboxaldehyde and 5methylfurfural (Table 1). Similar results were previously observed (V. Pereira et al., 2011), showing that HMF content in aged wines is extremely dependent of the heating temperature (above $50{ }^{\circ} \mathrm{C}$ ) and sugar content. Two furans exceeded their odour threshold in wines baked at $70^{\circ} \mathrm{C}$ (Table 1), 5-ethoxymethylfurfural (Oth of $90 \mu \mathrm{g} / \mathrm{L}$, (Cutzach, Chatonnet, \& Dubourdieu, 2000)) and HMF (Oth of $12 \mathrm{mg} / \mathrm{L}$, (Hauck, Landmann, Brühlmann, \& Schwab, 2003)). The former has surpassed 40 -fold the odour perception and the second only almost 2-fold. This result indicates that these furans may be potential odorants of baked Madeira wines. Indeed, Oliveira e Silva et al. (2008) observed that 5-ethoxymethylfurfural had an important role in the definition of the global aroma of baked Madeira wines. Cutzach et al. (1999) observed the same in sweet fortified wines and suggested that 5-ethoxymethylfurfural can be obtained by the reaction of HMF with ethanol, catalysed by the wine acidic medium and temperature.

Other minor furans, usually reported in aged wines (Cutzach et al., 1999; A. C. Pereira et al., 2010) were also identified, such as 2-acetylfuran, 2-acetyl-5-methylfuran, ethyl 2-furoate and furfuryl alcohol. Additionally, were also detected in baked wines minor furans rarely detected in wines, namely 2,2-dimethyl-5(1-methyl-1-propenyl)-tetrahydrofuran, dihydro-2-methyl-3 (2H)-furanone, 2-furyl ethyl ketone and 1-(5-hydroxymethyl-2furanyl)-1-propanone. 2,2-Dimethyl-5-(1-methyl-1-propenyl)-tetrahydrofuran has been detected in the volatile fraction of an ice wine (Setkova, Risticevic, \& Pawliszyn, 2007).

\subsection{Lactones}

Lactones are essentially formed by yeast during alcoholic fermentation, though significant odorant lactones are usually accu- mulated during wine ageing. The most common example is sotolon, which imparts powerful nutty, sweet, burnt, curry notes to those wines in which it is present. Oliveira e Silva et al. (2008) confirmed the importance of sotolon in the typicality of Madeira wines and observed a progressive increase of sotolon in Madeira wines submitted to estufagem. However, in the current baked Madeiras, this lactone was not detected, probably because the used analytical procedure was not appropriate to detect this lactone. Nonetheless, 7 lactones were currently detected, most of them never surpassing their odour perception. The exception was $\gamma$-decalactone, which slightly surpassed its low flavour threshold $(2.6 \mu \mathrm{g} / \mathrm{L}$, (Czerny et al., 2008)) in TN dry wine. The results also showed that this lactone, as well as most lactones, suffered a slight increase after the heating step. Quantitatively, the most abundant lactone was $\gamma$-carboethoxy- $\gamma$-butyrolactone, with values ranging between 79.9 and $539.3 \mu \mathrm{g} / \mathrm{L}$. Its content was higher in TN dry wine indicating that longer fermentations favour its formation. Additionally, the results also showed that $\gamma$-carboethoxy- $\gamma$-butyrolactone greatly increased after heating, indicating that temperature favours its formation. This lactone was already reported in Madeira wines aged in oak casks (A. C. Pereira et al., 2010). $\gamma$-Butyrolactone, commonly considered the most abundant wine lactone, since it is essentially derived from the fermentative process, was also detected with values ranging between 13.0 and $44.3 \mu \mathrm{g} / \mathrm{L}$. $\gamma$-Ethoxybutyrolactone was also detected, probably formed by the reaction between ethanol and $\gamma$-butyrolactone. This lactone was previously identified in the volatile fraction of toasted wood chips (Fernández de Simón, Esteruelas, Mun 0z, Cadahía, \& Sanz, 2009). Other minor lactones were also detected, namely $\gamma$-nonalactone and the two isomers ( $\alpha$ and $\beta$ ) of angelica lactone. Pisarnitskii (2001) has suggested that angelica lactones in wines are products of sugar decomposition. Actually, these lactones were only detected in sweet wines baked at overheating conditions.

\subsection{Sulfur compounds}

Volatile sulfur compounds in wines are usually responsible for unpleasant odours, even when present in trace amounts, since they possess low perception thresholds. Usually, their presence in wines is associated with the enzymatic (fermentative process) or nonenzymatic (storage or maturation processes) degradation of sulfur-containing compounds, especially amino acids (Mestres, Busto, \& Guasch, 2000). In the current wines only 4 sulfur compounds were encountered and all apparently below their odour perception limit. The volatile profile of TN sweet wine did not show any sulfur compounds, even after estufagem. Methionol was the most abundant sulfur compound, especially in TN dry wine (135.4-161.9 $\mu \mathrm{g} / \mathrm{L})$. In fact, TN dry wine accumulated higher contents, probably because it was more fermented. Fedrizzi et al. (2011) also found out that methionol was the most abundant sulfur-containing aroma compound. Besides methionol, ethyl 3(methylthio)propionate was also detected (3.1-17.4 $\mu \mathrm{g} / \mathrm{L}$ ). Both compounds are usually considered products of methionine metabolism (Mestres et al., 2000). 4-(Methylthio)-1-butanol, usually related with homomethionine metabolism (Mestres et al., 2000), was only found in TN dry wine (7.2-8.5 $\mu \mathrm{g} / \mathrm{L}$ ), but its tendency during estufagem could not be clearly established. According to the results of Fedrizzi et al. (2011), this compound was the second most abundant sulfur compound and has the tendency to decline under oxidative conditions. 2-Methyl-3-thiolanone, already identified in other wines (Aznar, López, Cacho, \& Ferreira, 2001), was also encountered in the current Madeira wines, with values ranging from 4.9 to $12.5 \mu \mathrm{g} / \mathrm{L}$. Generally speaking, it seems that the heating process did not promote significant changes in the levels of sulfur compounds. Fedrizzi et al. (2011) found out the depletion of most 
sulfur compounds after the first 15 days of oxidative ageing of Amarone wines.

\subsection{Acetals}

The origin of acetals in wines is usually associated with the reaction of aldehydes with hydroxyl groups of two alcohols. Madeira wines present favourable conditions for their production, since the alcoholic content is high and several aldehydes are present. In fact, 8 acetals were detected in the current sample set.

Heterocyclic acetals were also encountered in the current Madeiras, specifically cis-5-hydroxy-2-methyl-1,3-dioxane (cisdioxane), cis-4-hydroxymethyl-2-methyl-1,3-dioxolane (cis-dioxolane) and trans-5-hydroxy-2-methyl-1,3-dioxane (trans-dioxane). Interestingly, cis-dioxane always increased when the baking was conducted at standard conditions, but not always increased when the heating took place at higher temperatures. Similar trends were usually observed for cis-dioxolane and trans-dioxane.

Four diethyl acetals were also identified, namely the diethyl acetals of isovaleraldehyde, glycolaldehyde, furfural and phenylacetaldehyde, with values ranging from 2.0 to $20.4 \mu \mathrm{g} / \mathrm{L}$. Additionally, acetaldehyde ethyl amyl acetal, probably originated from the reaction of acetaldehyde with ethanol and amyl alcohol (1-pentanol), was also encountered. The acyclic acetals did not present a clear trend with the heating process.

\subsection{Other compounds}

Finally, 10 compounds that do not belong to the aforementioned chemical families were also found in the current Madeiras. Among them were 4 phthalates, which can be transferred to wines during winemaking from plastic materials (Carrillo, Salazar, Moreta, \& Tena, 2007). In general, phthalates concentration decreased after the thermal processing.

Another compound that is often reported in wines (Oliveira, Oliveira, Baumes, \& Maia, 2008; Perestrelo et al., 2006), N-(3-methylbutyl)acetamide, was also found, especially in TN dry wine. Other compounds were only developed after heating, which was the case of the following compounds, tentatively identified by mass spectra (comparison with NIST and Wiley libraries): 3,7-dimethyl-2, 3-epoxy-6-octanyl-1-oxythiocarbonylimidazolide, ethyl pyrrole2-carboxylate, 2,3-dihydroxypyrazine, curvulol and ethyl 2-formylpyrrole-1-acetate. Interestingly, the compound identified as 3,7-dimethyl-2,3-epoxy-6-octanyl-1-oxythiocarbonylimidazolide sharply increased after the thermal processing, from not detected up to $1351.7 \mu \mathrm{g} / \mathrm{L}$. Its increase was especially high in sweet wines.

\section{Conclusions}

The current study showed that estufagem introduced significant changes in the volatile composition of Madeira wines. The heating process promoted the increase of the volatile fraction regardless of the sweetness degree, especially increasing the complexity of sweet wines up to $88 \%$. Apparently, major differences were not observed in the volatile profile of wines obtained from different grape varieties after estufagem, although it was noticeable that before this step Malvasia was more abundant and richer than Tinta Negra. Moreover, the temperature increase (overheating experiment) had a great impact on the development of new volatile compounds, especially in sweet wines.

At least 190 volatile compounds were identified, 53 of which exclusively encountered in baked wines. It was observed that most chemical families increased after the heating step, especially furan compounds and esters. Thus, in quantitative terms, the volatile profile of baked Madeira wines was mostly represented by esters (more than 38\%) and alcohols (more than 15\%). Moreover, furan compounds (sugar by-products) play a significant role in the volatile fraction of sweet wines, especially when the heating is performed at high temperatures. Interestingly, 6 norisoprenoids were developed during baking, particularly $\beta$-damascenone, which exceeded the limit of olfactory perception, at least 70 -fold more in standard heating. In opposition, alcohols and fatty acids presented a slight decrease after heating. Similarly, most acetates declined after baking contributing to the loss of fruitiness. Additionally, several varietal aromas, such as monoterpenic alcohols, usually related to the floral character of some wines and especially encountered in Malvasia wine, were not detected after baking.

In terms of odorant impact, the obtained results showed that estufagem favoured the development of some volatiles previously reported as typical aromas of Madeira wines, particularly phenylacetaldehyde, $\beta$-damascenone and 5-ethoxymethylfurfural. Additionally, ethyl butyrate, ethyl 2-methylbutyrate, ethyl hexanoate, ethyl isovalerate, guaiacol, HMF and $\gamma$-decalactone were also found as potential contributors to the global aroma of baked wines.

\section{Acknowledgments}

Vanda Pereira acknowledges the Portuguese Fundação para a Ciência e Tecnologia for the financial support through the PhD grant SFRH/BD/24177/2005. The authors are also thankful to Madeira Wine Company for kindly supplying the wine samples used in this work.

\section{References}

Accordini, D. (2013). Amarone. In Sweet, reinforced and fortified wines (pp. 187-203). John Wiley \& Sons Ltd.

Ames, J. M., Guy, R. C. E., \& Kipping, G. J. (2001). Effect of pH, temperature, and moisture on the formation of volatile compounds in glycine/glucose model systems. Journal of Agricultural and Food Chemistry, 49, 4315-4323.

Aznar, M., López, R., Cacho, J. F., \& Ferreira, V. (2001). Identification and quantification of impact odorants of aged red wines from Rioja. GC-Olfactometry, quantitative GC-MS, and odor evaluation of HPLC fractions. Journal of Agricultural and Food Chemistry, 49, 2924-2929.

Baltes, W., \& Mevissen, L. (1988). Model reactions on roast aroma formation. Zeitschrift für Lebensmitteluntersuchung und-Forschung A, 187, 209-214.

Benítez, P., Castro, R., Natera, R., \& Barroso, C. (2006). Changes in the polyphenolic and volatile content of "Fino" Sherry wine exposed to high temperature and ultraviolet and visible radiation. European Food Research and Technology, 222, 302-309.

Campo, E., Ferreira, V., Escudero, A., Marques, J. C., \& Cacho, J. (2006). Quantitative gas chromatography-olfactometry and chemical quantitative study of the aroma of four Madeira wines. Analytica Chimica Acta, 563, 180-187.

Carrillo, J. D., Salazar, C. Moreta, C., \& Tena, M. T. (2007). Determination of phthalates in wine by headspace solid-phase microextraction followed by gas chromatography-mass spectrometry: Fibre comparison and selection. Journal of Chromatography A, 1164, 248-261.

Cejudo-Bastante, M. J.. Hermosín-Gutiérrez, I., \& Pérez-Coello, M. S. (2013). Accelerated aging against conventional storage: Effects on the volatile composition of Chardonnay white wines. Journal of Food Science, 78, C507-C513.

Cutzach, I., Chatonnet, P., \& Dubourdieu, D. (1999). Study of the formation mechanisms of some volatile compounds during the aging of sweet fortified wines. Journal of Agricultural and Food Chemistry, 47, 2837-2846.

Cutzach, I., Chatonnet, P., \& Dubourdieu, D. (2000). Influence of storage conditions on the formation of some volatile compounds in white fortified wines (Vins doux Naturels) during the aging process. Journal of Agricultural and Food Chemistry, 48, 2340-2345.

Czerny, M., Christlbauer, M., Christlbauer, M., Fischer, A., Granvogl, M., Hammer, M. Hartl, C., Hernandez, N., \& Schieberle, P. (2008). Re-investigation on odour thresholds of key food aroma compounds and development of an aroma language based on odour qualities of defined aqueous odorant solutions. European Food Research and Technology, 228, 265-273.

deMan, J. M. (1999). Additives and contaminants. In J. M. deMan (Ed.), Principles of food chemistry (3rd ed., pp. 429-449). New York: Springer Science.

Ebeler, S. E. (2001). Analytical chemistry: Unlocking the secrets of wine flavor. Food Reviews International, 17, 45-64.

Escudero, A., Cacho, J., \& Ferreira, V. (2000). Isolation and identification of odorants generated in wine during its oxidation: A gas chromatography-olfactometric study. European Food Research and Technology, 211, 105-110.

Fedrizzi, B., Zapparoli, G., Finato, F., Tosi, E., Turri, A., Azzolini, M., \& Versini, G. (2011). Model aging and oxidation effects on varietal, fermentative, and sulfur 
compounds in a dry botrytized red wine. Journal of Agricultural and Food Chemistry, 59, 1804-1813.

Fernández de Simón, B. g., Esteruelas, E., Mun oz, A. n. M., Cadahía, E., \& Sanz, M. (2009). Volatile compounds in acacia, chestnut, cherry, ash, and oak woods, with a view to their use in cooperage. Journal of Agricultural and Food Chemistry, 57, 3217-3227.

Flamini, R., \& Traldi, P. (2010). Grape aroma compounds: Terpenes, C13norisoprenoids, benzene compounds, and 3-alkyl-2-methoxypyrazines. In R. Flamini \& P. Traldi (Eds.), Mass spectrometry in grape and wine chemistry (pp. 95-116). Hoboken: John Wiley \& Sons Inc.

Gómez-Míguez, M. J., Cacho, J. F., Ferreira, V., Vicario, I. M., \& Heredia, F. J. (2007). Volatile components of Zalema white wines. Food Chemistry, 100, 1464-1473.

Güntert, M., Rapp, A., Takeoka, G. R., \& Jennings, W. (1986). HRGC and HRGC-MS applied to wine constituents of lower volatility. Zeitschrift für Lebensmitteluntersuchung und-Forschung A, 182, 200-204.

Hauck, T., Landmann, C., Brühlmann, F., \& Schwab, W. (2003). Formation of 5methyl-4-hydroxy-3[2H]-furanone in cytosolic extracts obtained from Zygosaccharomyces rouxii. Journal of Agricultural and Food Chemistry, 51, 1410-1414.

Janusz, A., Capone, D. L., Puglisi, C. J., Perkins, M. V., Elsey, G. M., \& Sefton, M. A. (2003). (E)-1-(2,3,6-Trimethylphenyl)buta-1,3-diene: A potent grape-derived odorant in wine. Journal of Agricultural and Food Chemistry, 51, 7759-7763.

Kanasawud, P., \& Crouzet, J. C. (1990). Mechanism of formation of volatile compounds by thermal degradation of carotenoids in aqueous medium. 1 beta.-Carotene degradation. Journal of Agricultural and Food Chemistry, 38, $237-243$.

Ledauphin, J., Saint-Clair, J.-F., Lablanquie, O., Guichard, H., Founier, N., Guichard, E., \& Barillier, D. (2004). Identification of trace volatile compounds in freshly distilled Calvados and Cognac using preparative separations coupled with gas chromatography-mass spectrometry. Journal of Agricultural and Food Chemistry, 52, 5124-5134.

Lee, S.-J., \& Noble, A. C. (2003). Characterization of odor-active compounds in Californian Chardonnay wines using GC-Olfactometry and GC-Mass Spectrometry. Journal of Agricultural and Food Chemistry, 51, 8036-8044.

López, R., Aznar, M., Cacho, J., \& Ferreira, V. (2002). Determination of minor and trace volatile compounds in wine by solid-phase extraction and gas chromatography with mass spectrometric detection. Journal of Chromatography A, 966, 167-177.

López de Lerma, N., Peinado, J., Moreno, J., \& Peinado, R. A. (2010). Antioxidant activity, browning and volatile Maillard compounds in Pedro Ximénez sweet wines under accelerated oxidative aging. LWT - Food Science and Technology, 43, 1557-1563.

Loscos, N., Hernández-Orte, P., Cacho, J., \& Ferreira, V. (2010). Evolution of the aroma composition of wines supplemented with grape flavour precursors from different varietals during accelerated wine ageing. Food Chemistry, 120, 205-216.

Marais, J. (1983). Terpenes in the aroma of grapes and wines: A review. South African Journal of Enology and Viticulture, 4, 49-58.

Mestres, M., Busto, O., \& Guasch, J. (2000). Analysis of organic sulfur compounds in wine aroma. Journal of Chromatography A, 881, 569-581.

Nebesny, E., Budryn, G., Kula, J., \& Majda, T. (2007). The effect of roasting method on headspace composition of robusta coffee bean aroma. European Food Research and Technology, 225, 9-19.

Nykanen, L. (1986). Formation and occurrence of flavor compounds in wine and distilled alcoholic beverages. American Journal of Enology and Viticulture, 37, 84-96.
Oliveira e Silva, H., Guedes de Pinho, P., Machado, B. P., Hogg, T., Marques, J. C. Câmara, J. S., Albuquerque, F., \& Silva Ferreira, A. C. (2008). Impact of forcedaging process on Madeira wine flavor. Journal of Agricultural and Food Chemistry, 56, 11989-11996.

Oliveira, J. M., Oliveira, P., Baumes, R. L., \& Maia, O. (2008). Changes in aromatic characteristics of Loureiro and Alvarinho wines during maturation. Journal of Food Composition and Analysis, 21, 695-707.

Pereira, V. (2011). Effect of the estufagem process on the chemical constituents of Madeira wines. Unpublished Ph.D. thesis, University of Madeira, Funchal.

Pereira, V., Albuquerque, F. M., Ferreira, A. C., Cacho, J., \& Marques, J. C. (2011) Evolution of 5-hydroxymethylfurfural (HMF) and furfural (F) in fortified wines submitted to overheating conditions. Food Research International, 44, 71-76.

Pereira, A. C., Reis, M. S., Saraiva, P. M., \& Marques, J. C. (2010). Analysis and assessment of Madeira wine ageing over an extended time period through GCMS and chemometric analysis. Analytica Chimica Acta, 660, 8-21.

Perestrelo, R., Fernandes, A., Albuquerque, F. F., Marques, J. C., \& Câmara, J. S. (2006). Analytical characterization of the aroma of Tinta Negra Mole red wine: Identification of the main odorants compounds. Analytica Chimica Acta, 563, 154-164.

Pisarnitskii, A. F. (2001). Formation of wine aroma: Tones and imperfections caused by minor components. Applied Biochemistry and Microbiology, 37, 552-560.

Polaskova, P., Herszage, J., \& Ebeler, S. E. (2008). Wine flavor: Chemistry in a glass. Chemical Society Reviews, 37, 2478-2489.

Sánchez-Palomo, E., Gómez García-Carpintero, E., Alonso-Villegas, R., \& GonzálezViñas, M. A. (2010). Characterization of aroma compounds of Verdejo white wines from the La Mancha region by odour activity values. Flavour and Fragrance Journal, 25, 456-462.

Schneider, R., Baumes, R., Bayonove, C., \& Razungles, A. (1998). Volatile compounds involved in the aroma of sweet fortified wines (Vins doux Naturels) from Grenache Noir. Journal of Agricultural and Food Chemistry, 46, 3230-3237.

Setkova, L., Risticevic, S., \& Pawliszyn, J. (2007). Rapid headspace solid-phase microextraction-gas chromatographic-time-of-flight mass spectrometric method for qualitative profiling of ice wine volatile fraction: II: Classification of Canadian and Czech ice wines using statistical evaluation of the data. Journal of Chromatography A, 1147, 224-240.

Silva Ferreira, A. C., Barbe, J.-C., \& Bertrand, A. (2003). 3-Hydroxy-4,5-dimethyl$2(5 \mathrm{H})$-furanone: A key odorant of the typical aroma of oxidative aged Port wine Journal of Agricultural and Food Chemistry, 51, 4356-4363.

Soares da Costa, M., Gonçalves, C., Ferreira, A., Ibsen, C., Guedes de Pinho, P., \& Silva Ferreira, A. C. (2004). Further insights into the role of methional and phenylacetaldehyde in lager beer flavor stability. Journal of Agricultural and Food Chemistry, 52, 7911-7917.

Stein, S. E. (2008). NIST Chemistry WebBook, NIST Standard Reference Database Number 69. In P. J. L. a. W. G. Mallard (Ed.), Gas chromatography (vol. 2011). Gaithersburg MD: National Institute of Standards and Technology.

Tat, L., Comuzzo, P., Battistutta, F., \& Zironi, R. (2007). Sweet-like off-flavor in Aglianico del Vulture wine: Ethyl phenylacetate as the mainly involved compound. Journal of Agricultural and Food Chemistry, 55, 5205-5212.

Versini, G., Dellacassa, E., Carlin, S., Fedrizzi, B., \& Magno, F. (2008). Analysis of aroma compounds in wine. In R. Flamini (Ed.), Hyphenated techniques in grape and wine chemistry (pp. 173-225). Chichester: John Wiley \& Sons Ltd. 